\title{
Frontal theta and posterior alpha in resting EEG: A critical examination of convergent and discriminant validity
}

\author{
Ezra E. Smith ${ }^{1}$ (D) | Craig E. Tenke ${ }^{1,2,3}$ | Patricia J. Deldin ${ }^{4}$ | Madhukar H. Trivedi $^{5}$ | \\ Myrna M. Weissman $^{1,2}$ | Randy P. Auerbach ${ }^{2}$ | Gerard E. Bruder ${ }^{2}$ | \\ Diego A. Pizzagalli $^{6,7}$ | Jürgen Kayser ${ }^{1,2,3}$ (D)
}

${ }^{1}$ Division of Translational Epidemiology, New York State Psychiatric Institute, New York, New York, USA

${ }^{2}$ Department of Psychiatry, Vagelos College of Physicians \& Surgeons, Columbia University, New York, New York, USA

${ }^{3}$ Division of Cognitive Neuroscience, New York State Psychiatric Institute, New York, New York, USA

${ }^{4}$ Department of Psychiatry, University of Michigan, Ann Arbor, Michigan, USA

${ }^{5}$ Department of Psychiatry, University of Texas, Southwestern Medical Center, Dallas, Texas, USA

${ }^{6}$ Department of Psychiatry, Harvard Medical School, Boston, Massachusetts, USA

${ }^{7}$ Center for Depression, Anxiety \& Stress Research, McLean Hospital, Belmont, Massachusetts, USA

\section{Correspondence}

Jürgen Kayser, Division of Translational Epidemiology, New York State Psychiatric Institute, Unit 50, 1051 Riverside Drive, New York, NY 10032, USA.

Email: jurgen.kayser@nyspi.columbia.edu

Funding information

National Institute of Mental Health, Grant/ Award Number: MH092221, MH092250 and MH115299

\begin{abstract}
Prior research has identified two resting EEG biomarkers with potential for predicting functional outcomes in depression: theta current density in frontal brain regions (especially rostral anterior cingulate cortex) and alpha power over posterior scalp regions. As little is known about the discriminant and convergent validity of these putative biomarkers, a thorough evaluation of these psychometric properties was conducted toward the goal of improving clinical utility of these markers. Resting 71-channel EEG recorded from 35 healthy adults at two sessions (1-week retest) were used to systematically compare different quantification techniques for theta and alpha sources at scalp (surface Laplacian or current source density [CSD]) and brain (distributed inverse; exact low resolution electromagnetic tomography [eLORETA]) level. Signal quality was evaluated with signal-to-noise ratio, participant-level spectra, and frequency PCA covariance decomposition. Convergent and discriminant validity were assessed within a multitrait-multimethod framework. Posterior alpha was reliably identified as two spectral components, each with unique spatial patterns and condition effects (eyes open/closed), high signal quality, and good convergent and discriminant validity. In contrast, frontal theta was characterized by one lowvariance component, low signal quality, lack of a distinct spectral peak, and mixed validity. Correlations between candidate biomarkers suggest that posterior alpha components constitute reliable, convergent, and discriminant biometrics in healthy adults. Component-based identification of spectral activity (CSD/eLORETA-fPCA) was superior to fixed, a priori frequency bands. Improved quantification and conceptualization of frontal theta is necessary to determine clinical utility.
\end{abstract}

\section{K E Y W O R D S}

current source density (CSD), EEG biomarkers, frequency PCA, source localization (LORETA), theta/ alpha oscillations, validity

\section{1 | INTRODUCTION}

Identifying disease-specific biomarkers that elucidate pathophysiology and predict treatment outcomes is a key target for clinical neuroscience. Although there has been significant progress in this regard (Kühn et al., 2009; Light et al., 2015; Monti et al., 2010; Pizzagalli et al., 2018; Weinberg, Riesel, \& Hajcak, 2012), methodological heterogeneity (i.e., 
identification and quantification of biomarkers) presents a significant barrier to cross-study comparison, interpretation of results, replicability, and clinical application. For major depressive disorder (MDD), several EEG biomarkers have shown promise for predicting treatment outcomes (for reviews, see Bruder, Tenke, \& Kayser, 2013; Iosifescu, 2011; Widge et al., 2018). Two candidate biomarkers that rely on resting EEG activity are currently under investigation as part of a large multisite study (Trivedi et al., 2016): rostral anterior cingulate cortex (rACC) theta, identified via a current density distributed inverse solution (low resolution brain electromagnetic tomography [LORETA]; e.g., Pizzagalli et al., 2001, 2018) and posterior alpha, identified via scalp current source density (CSD; surface Laplacian) and frequency principal component analysis (fPCA; e.g., Tenke et al., 2011). These two biomarkers have demonstrated moderate predictive validity (Pizzagalli et al., 2018; Tenke et al., 2011; Widge et al., 2018), but, in general, published results on EEG biomarkers are biased toward small studies with large effect sizes and positive results (Widge et al., 2018). Moreover, there is a notable absence of research evaluating techniques for biomarker quantification, discriminant validity between biomarkers, and convergent validity across methodologies. This study aimed to investigate questions regarding quantification and validity of these two candidate biomarkers.

\section{1 | Research findings on rACC theta as a candidate biomarker}

There is an ongoing effort to identify biomarkers that predict which depressed patients will improve following treatment (e.g., Pizzagalli, 2011; Waters \& Mayberg, 2017). Among those, greater theta-band activity in the rACC has been repeatedly linked to favorable clinical outcome (Pizzagalli, 2011; Pizzagalli et al., 2001, 2018). Specifically, enhanced theta magnitude in the rACC, and also when measured at frontal scalp locations (i.e., midfrontal theta [MF $\theta]$ ), predicted symptom remission of MDD patients who received antidepressant medications (e.g., selective serotonin reuptake inhibitors [SSRIs]; Hunter, Korb, Cook, \& Leuchter, 2013; Korb, Hunter, Cook, \& Leuchter, 2009; Mulert et al., 2007; Pizzagalli et al., 2001, 2018; Rentzsch, Adli, Wiethoff, Gómez-Carrillo de Castro, \& Gallinat, 2014), noninvasive (i.e., transcranial magnetic stimulation; Li et al., 2016) or invasive neuromodulation (i.e., implantation of a deep-brain stimulator; Broadway et al., 2012), and also of those assigned to a placebo control group (Pizzagalli et al., 2018). These findings suggest that frontal/rACC theta may be a nonspecific predictor of symptom improvement in individuals with MDD. It is hypothesized that individuals with low rACC theta may have difficulty with cognitive control, and consequently these individuals are more likely to have a poor clinical prognosis (e.g., Mayberg, 1997; Pizzagalli,
2011; Waters \& Mayberg, 2017). Notably, two recent multisite studies reported contradictory results. Pizzagalli et al. (2018) found a positive relationship between pretreatment rACC theta and symptom improvement in a large sample of depressed patients $(N=248)$, indicating that MDD patients with more pretreatment rACC theta were more likely to improve after 8 weeks regardless of treatment arm (placebo vs. SSRI; for additional independent replications, see Korb et al., 2009; Mulert et al., 2007; Pizzagalli et al., 2001; Rentzsch et al., 2014). In contrast, employing an even larger sample of MDD patients ( $N=1,008)$, Arns et al. (2015) found that less pretreatment rACC theta was associated with improvement, that is, a relationship between rACC theta and symptom change opposite the expected direction. These findings prompted us to examine whether different methods of rACC theta quantification could explain these inconsistencies.

Many reports have noted that frontal theta activity is weak and infrequent in resting EEG recordings of wakeful participants (e.g., Barry \& De Blasio, 2018; Cigánek, 1961; Frauscher et al., 2018; Keitel \& Gross, 2016; Schacter, 1977; Shackman, McMenamin, Maxwell, Greischar, \& Davidson, 2010; Tenke \& Kayser, 2005; Westmoreland \& Klass, 1986), meaning that there is no continuous or dominant theta rhythm except for limited bursts that can be obscured by fast Fourier transform (FFT) averaging (e.g., Vidaurre et al., 2018). This evidence indicates that only a fraction of resting EEG recordings contain notable oscillations below $8 \mathrm{~Hz}$ in wakeful resting participants (Arns, Gordon, \& Boutros, 2017; Keitel \& Gross, 2016; Schacter, 1977; Vidaurre et al., 2018; Westmoreland \& Klass, 1986). In fact, frontal theta is inversely related to default-mode network activity (Scheeringa et al., 2008). When theta is apparent in resting EEG, it is often associated with greater drowsiness and ocular artifact (Mcmenamin et al., 2010; Schacter, 1977; Strijkstra, Beersma, Drayer, Halbesma, \& Daan, 2003). Moreover, datadriven approaches (i.e., principal component analysis [PCA]) often fail to identify a spectral theta component in resting EEG recordings (Barry \& De Blasio, 2018; Shackman et al., 2010; Tenke \& Kayser, 2005). Variation in theta amplitude as a function of EEG hardware and personnel further underscores the subtlety of this metric (Tenke et al., 2017). Based on early factor analyses identifying functionally independent EEG frequency ranges (Kubicki et al., 1979), several studies have evaluated rACC theta as a candidate biomarker by quantifying spectral activity between 6.5 and $8.0 \mathrm{~Hz}$ (Arns et al., 2015; Mulert et al., 2007; Pizzagalli et al., 2001); however, this limited frequency range deviates from conventional definition of theta (Chatrian et al., 1974; Kane et al., 2017), and it may also be more prone to assessing a mixture of theta and alpha oscillations. What may ultimately be even more important is that a dominating theta rhythm, which is observed during task performance involving working memory load or 
cognitive control, peaks at $5 \mathrm{~Hz}$ (e.g., Cavanagh \& Shackman, 2015). Phasic and induced theta oscillations are prominent at $5 \mathrm{~Hz}$ over midfrontal regions and are consistently related to cognitive and behavioral performance (Cohen \& van Gaal, 2014; Hsieh \& Ranganath, 2014; Li et al., 2016; Näpflin, Wildi, \& Sarnthein, 2008; Olvet \& Hajcak, 2009; Schacter, 1977). To the extent that this evoked $5 \mathrm{~Hz}$ rhythm is directly related to resting theta, its peak frequency will be missed by a $6.5-8.0 \mathrm{~Hz}$ band quantification.

Moreover, although the $\mathrm{rACC}$ is the typical region of interest (ROI) for measuring resting-state theta, dorsal and posterior ACC (dACC and PCC) regions have demonstrated stronger resting theta. For example, Li and colleagues (2016) found that theta amplitude at rest correlated with glucose uptake in the dACC, whereas theta oscillations induced by cognitive testing correlated with glucose uptake in the rACC. Although the logic of focusing on the rACC as a region critical for depression was driven by early positron emission tomography (PET) findings of Mayberg et al. (1997), later confirmed by Pizzagalli et al. (2001) using EEG source localization, more recent evidence suggests that theta generators outside the rACC may be more potent indicators of theta, and these regions are closely linked to psychological constructs relevant to treatment response (Cavanagh \& Shackman, 2015). In particular, a magnetoencephalogram (MEG) study reported higher testretest reliability of resting theta for dorsal-posterior ACC than rACC (Martín-Buro, Garcés, \& Maestú, 2016), and an intracranial EEG study revealed strong theta sources near dorsal ACC regions at rest (Frauscher et al., 2018). Notably, an exploratory whole-brain analysis by Arns and colleagues (2015) found that treatment response correlated with less theta amplitude near the $\mathrm{dACC}$, rather than the rACC, suggesting that the dACC may also be relevant to treatment outcome. Therefore, refocusing analyses toward stronger theta generators (e.g., dACC) could improve measurement quality and clinical prediction.

Another important consideration is the impact of computational procedures intended to improve the signal of interest. One often-used optimization technique - spatial normalization-actually mixes theta activity from different brain regions and could impede high-fidelity measurement and confuse spatial interpretation. Spatial normalization is calculated by dividing theta current density in the rACC by the sum of theta current density across the entire brain (e.g., Pizzagalli et al., 2003; Smith, Cavanagh, \& Allen, 2018). Thus, the relationship between normalized $\mathrm{ACC}$ theta and MDD recovery may result from more $\mathrm{ACC}$ theta, less theta elsewhere in the brain (e.g., PCC), or both. Notably, studies not relying on spatial normalization found that less frontal theta (Arns et al., 2015; Iosifescu et al., 2009; Knott, Telner, Lapierre, Browne, \& Horn, 1996; Leuchter et al., 2017) and more PCC theta (Arns et al., 2015) predicted clinical improvement.

\subsection{Research findings on posterior alpha- band activity as a candidate biomarker}

In comparison to resting theta oscillations, the alpha rhythm dominates the resting EEG, with most individuals showing a distinct alpha peak at about $10 \mathrm{~Hz}$ having a robust posterior topography (Aurlien et al., 2004; Chiang, Rennie, Robinson, van Albada, \& Kerr, 2011). Moreover, the alpha rhythm is prominent (e.g., visible in raw EEG traces) and reliably quantified by different research groups using different methodologies (e.g., Barry \& De Blasio, 2018; Labounek et al., 2018; Schmidt et al., 2017; Shackman et al., 2010; Sockeel, Schwartz, Pélégrini-issac, \& Benali, 2016; Tenke et al., 2017). Importantly, greater posterior alpha oscillations at rest predicted a favorable clinical outcome for individuals diagnosed with MDD (Baskaran et al., 2017; Bruder et al., 2008; Jaworska, de la Salle, Ibrahim, Blier, \& Knott, 2019; Kandilarova et al., 2017; Knott et al., 1996; Tenke et al., 2011; Ulrich, Renfordt, Zeller, \& Frick, 1984; Ulrich, Renfordt, \& Frick, 1986; although see Arns et al., 2016, and Knott, Mahoney, Kennedy, \& Evans, 2000, for unsuccessful attempts to replicate these findings). To some degree, these reports differed in methodology, including EEG montage (density, locations) and reference, preprocessing steps, and a priori selection of frequency bins. Notably, there are significant benefits from using EEG signal separation as a generic initial preprocessing step, especially with regard to improved accuracy of measuring brain activity from specific neural sources and empirical (i.e., data-driven) determination of optimal frequency bands and recording locations (Cohen, 2017a; Delorme \& Makeig, 2004; Tenke \& Kayser, 2005). For example, whereas spectral amplitude within an approximate range of $8-13 \mathrm{~Hz}$ is often averaged for a pooled analysis of the (classical) alpha band, narrower bands in this range have distinct functional significance (Buzsáki, 2006; Klimesch, 1999; Sadaghiani \& Kleinschmidt, 2016) and spatial distribution (Barzegaran, Vildavski, \& Knyazeva, 2017; Tenke \& Kayser, 2005). Specifically, Tenke and colleagues demonstrated the presence of two distinct spectral alpha components, ${ }^{1}$ peaking at around 9 and $11 \mathrm{~Hz}$, with $9 \mathrm{~Hz}$ component loadings crossing over to the classical theta band (i.e., well below $8 \mathrm{~Hz}$ ), suggesting that low alpha and theta activity share variance (e.g., Tenke et al., 2011, termed this spectral component low alpha/theta). Indeed, fPCA applied to resting-state EEG has consistently revealed two distinct alpha components: one low alpha component with an $\sim 9 \mathrm{~Hz}$ peak frequency and a lateral occipitoparietal topography and another high alpha component peaking at $\sim 11 \mathrm{~Hz}$ with an occipitoparietal midline maximum (Barry \& De Blasio, 2018; Barzegaran et al., 2017; Chiang et al., 2011; Shackman et al., 2010; Tenke \& Kayser, 2005). As "true" rACC theta variance may be weak at rest, it stands to

\footnotetext{
${ }^{1}$ Throughout the article, component refers to a spectral component
} identified by fPCA. 
reason that $6.5-8 \mathrm{~Hz}$ spatially normalized rACC activity, which is influenced by posterior activity, may partly be measuring a posterior alpha covariance entity. Moreover, spectral windows within fixed frequency limits do not actually separate genuine EEG rhythms (i.e., theta and alpha) because spectral leakage conflates neighboring bands. Taken together, various suboptimal methodological choices may conflate theta and alpha metrics by mixing variance sources. Hence, the different specific techniques used to calculate these biomarkers should be directly compared.

\section{3 | Present report}

This report aimed to systematically compare the effects of processing choices for the quantification and validity of resting EEG biomarkers. An evaluation of discriminant and convergent validity can reveal the extent to which biomarkers are robust to specific methodologies and to what extent biomarkers are distinct from one another (Campbell \& Fiske, 1959). Although decisions regarding EEG methodology are often arbitrary or based on a priori hypotheses (e.g., a priori frequency bands, EEG reference, ROIs, spatial normalization), we argue that in many cases data-driven approaches will outperform investigator-guided processing choices. Datadriven approaches will reduce unsystematic nuisance variance in EEG data (e.g., Barry \& De Blasio, 2018; Delorme, Palmer, Onton, Oostenveld, \& Makeig, 2012; Kayser, Tenke, \& Debener, 2000; Tenke \& Kayser, 2005). Hence, frequency analyses in this report were guided by an unbiased extraction of spectral components (i.e., multivariate data decomposition via fPCA; Kayser et al., 2000; Tenke \& Kayser, 2005; Tenke et al., 2011). fPCA identifies spectral components from their covariance structure across conditions, electrodes, and/or participants. Most importantly, fPCA has proven to be a useful tool for the quantification of the latent structure of EEG spectra (e.g., Barry \& De Blasio, 2018; Shackman et al., 2010; Tenke \& Kayser, 2005).

Furthermore, ambiguity of reference choice is resolved, and spatial signal smearing due to volume conduction is mitigated, by transformation of scalp potentials into a referencefree representation of radial current flow (surface Laplacian or scalp CSD; Kayser \& Tenke, 2015b; Nunez \& Srinivasan, 2006; Tenke \& Kayser, 2012). Source modeling of resting EEG spectra also represents a reference-free approach, although this requires-in contrast to the CSD transformadditional biophysiological assumptions (e.g., Michel et al., 2004; Nunez, Nunez, \& Srinivasan, 2019; Pascual-Marqui, 2007). CSD and inverse models have the additional advantage of estimating possible locations of neural generator sources.

To this end, we analyzed current source estimates of EEG resting-state recordings at sensor-level (radial current flow via scalp CSD; Tenke \& Kayser, 2012, 2015b) and voxel-level (distributed inverses via exact low resolution electromagnetic tomography [eLORETA]; Pascual-Marqui, 2007) for theta and alpha oscillations in a sample of healthy adults (Tenke et al., 2017). We sought to identify cortical regions, particularly within cingulate cortex, where theta was maximal by taking signal-to-noise ratio (SNR) into account (Cohen, 2014b; Cohen \& Gulbinaite, 2017; Smith, Reznik, Stewart, \& Allen, 2017). Given previous work (Frauscher et al., 2018; Li et al., 2016; Martín-Buro et al., 2016), it was expected that dorsal and posterior cingulate regions would demonstrate greater SNR for the theta band compared to rACC and subgenual ACC (sgACC) regions. We also explored fPCA results for a frontal theta component, to facilitate identification of an optimal intracranial target to measure theta as well as optimal frequency band limits. A multitrait-multimethod framework (MTMM; Campbell \& Fiske, 1959) was used to assess discriminant and convergent validity for frontal theta and posterior alpha (see Bruder et al., 2013, and Pizzagalli, 2011, for reviews). For MTMM, patterns of correlations between methods and traits are interpreted qualitatively with respect to one another, with different patterns having implications for convergent and discriminant validity. MTMM correlations are descriptive measures of standardized covariance among indices and are not interpreted as inferential statistics. A MTMM analysis can elucidate construct validity and has the advantage of being technically parsimonious. We also applied fPCA to eLORETA distributed inverses (Barzegaran et al., 2017; Pascual-Marqui, 2007) as well as a combined data set using both CSD and eLORETA estimates.

Given prior research reviewed above, we anticipated poor discriminant validity between a posterior low-frequency (peaking around $9 \mathrm{~Hz}$ ) alpha component and spatially normalized rACC theta (using the typical $6.5-8 \mathrm{~Hz}$ band limits). In contrast, we anticipated good convergent validity between CSD and eLORETA spectral principal components, good discriminant validity between theta and alpha spectral components, and good test-retest reliability for spectral components. Overall, we anticipated that clarifying relationships between $\mathrm{rACC}$ theta and posterior alpha measures, which has not yet been systematically addressed in the literature, could aid the interpretation of each metric, help explain inconsistent findings across reports, and lead to improvements in EEG biomarker quantification and validity.

\section{2 | METHOD}

\section{1 | Participants}

Details regarding participant recruitment and selection are presented in Tenke et al. (2017). Briefly, a total of 35 healthy, English-fluent adults aged 18-65 years were tested 
as part of the Establishing Moderators and Biosignatures of Antidepressant Response in Clinical Care (EMBARC) project (Trivedi et al., 2016). Participants were locally recruited and tested at four sites: Columbia University Medical Center in New York, University of Texas Southwestern Medical Center in Dallas, Massachusetts General Hospital in Boston, and University of Michigan in Ann Arbor. As part of the larger randomized clinical trial EMBARC, these participants represented the subsample of healthy controls who were free of lifetime psychiatric disorder as assessed by an interviewer trained in the administration of the Structured Clinical Interview for DSM-IV Axis I Disorders, Nonpatient Edition (First, Gibbon, Spitzer, \& Williams, 1996). Each participant had two 8-min resting EEG sessions separated by about 1 week. Each EEG recording included four 2-min blocks with eyes closed (C) or open (O) in a fixed order (OCCO). Participants also completed a battery of biometric assays and self-report questionnaires that are not pertinent to this report. The study was conducted in accordance with the Declaration of Helsinki, was approved by the institutional review board at each testing site, and all participants provided informed consent.

\section{2 $\quad$ EEG processing}

All EEG pre- and postprocessing steps, including unification of EEG montage and acquisition parameters, have been detailed in Tenke et al. (2017). Briefly, data from the different research testing sites were converted to a common 72-channel EEG montage and visually inspected for recording artifacts. Missing, bad, or bridged channels (Alschuler, Tenke, Bruder, \& Kayser, 2014) were replaced by spherical spline interpolation (Perrin, Pernier, Bertrand, \& Echallier, 1989, 1990). The continuous EEG data were then blink corrected via spatial singular value decomposition and segmented into 2-s epochs with $75 \%$ overlap. Epochs were band-passed at 1-60 Hz (24 dB/octave). A semiautomated reference-free approach identified isolated EEG channels containing amplifier drift, residual eye activity, muscle or movement-related artifacts on a trial-bytrial basis (Kayser \& Tenke, 2006c). Channels containing artifact were replaced by spline interpolation if less than $25 \%$ of all channels were flagged; otherwise, the epoch was rejected. Finally, an automatic threshold $( \pm 100 \mu \mathrm{V})$ applied to all EEG and uncorrected electro-oculogram (EOG) channels removed any residual artifacts epoch-wise. The average total number of usable epochs for eyes open (EO) and eyes closed (EC) were 331.4 (range 191-467) and 377.7 (74-477) in Session 1, and 329.7 (90-480) and 373.6 (44-476) in Session 2.

To be consistent with the LORETA analysis previously employed using these data (Pizzagalli et al., 2018; Tenke et al., 2017), the nose site was excluded from these data for all analyses, including those at sensor level, rendering a 71channel montage. Average-referenced ${ }^{2}$ and epoched data were transformed to CSD (surface Laplacian; Nunez et al., 2019; Nunez \& Srinivasan, 2006; Tenke \& Kayser, 2012) and eLORETA representations (Pascual-Marqui, 2007) using previously recommended parameters for CSD (spline flexibility $m=4$, regularization constant $\lambda=10^{-5}$; e.g., Kayser, 2009; Kayser \& Tenke, 2006b; Tenke \& Kayser, 2005) or default parameters as implemented in the eLORETA software package (Pascual-Marqui, 2007). As the capacity of CSD estimates to represent different spatial frequencies is affected by choosing more (e.g., $m=3$ ) or less (e.g., $m=5$ ) flexible splines (Kayser \& Tenke, 2015b), their spectral quantification using fPCA (Tenke \& Kayser, 2005) may be affected as well. CSD spline flexibility affects the sensitivity of CSD measures to differentially represent broader versus more focal dipole layers (i.e., the spatial scale of the underlying neuronal generator sources; see fig. 15 in Kayser \& Tenke, 2015b). We evaluated the effect of six different spline flexibilities $(m=2 \ldots 7)$ on fPCA results. These analyses revealed that fPCA produced highly consistent spectral components regardless of spline flexibility (see online supporting information, Figures S1 and S2, for alpha and theta components, respectively). Accordingly, only CSD findings for a medium (default, $m=4$ ) spline flexibility are detailed here. The FFT was used for calculation of spectral amplitude from 2-s epochs (i.e., Tenke et al., 2017). CSD-based midfrontal theta (MF $\theta$; Table 1) was operationalized as the $6.5-8 \mathrm{~Hz}$ signal amplitude from the mean of sensors $\mathrm{FCz}$ and $\mathrm{Cz}$. Spatially normalized MF $\theta$ was calculated as the $6.5-8 \mathrm{~Hz}$ amplitude at a sensor divided by the sum of $6.5-8 \mathrm{~Hz}$ theta amplitudes across all scalp sensors.

\subsection{SNR and internal consistency}

Given that calculation of SNR is arbitrary with regard to what is considered signal and noise, we considered two different SNR calculations. In both cases, SNR was calculated directly from amplitude spectra rather than spectral components. One technique $\left(\mathrm{SNR}_{\mathrm{avg}}\right)$ defined $\mathrm{SNR}$ as the ratio of spectral amplitude at a single frequency bin to the average amplitude of the surrounding $\pm 5 \mathrm{~Hz}$ interval, excluding the $\pm 1 \mathrm{~Hz}$ around each bin (i.e., $\mathrm{SNR}_{\mathrm{avg}}[f]=f_{\mathrm{amp}} / \operatorname{mean}[f-3$ $\left.\left.\cdots f-1_{\mathrm{amp}}+f+1 \cdots f+3_{\mathrm{amp}}\right]\right)$. This approach characterizes subject-specific peaks in averaged amplitude spectra as having large SNR, and noise is assumed to be broadband or

\footnotetext{
${ }^{2}$ As only CSD and eLORETA transformed data were analyzed (i.e., no reference-dependent surface potentials), the actual reference choice is inconsequential. CSD and eLORETA are reference-free representations of EEG data and will produce the same unique result regardless of reference scheme.
} 


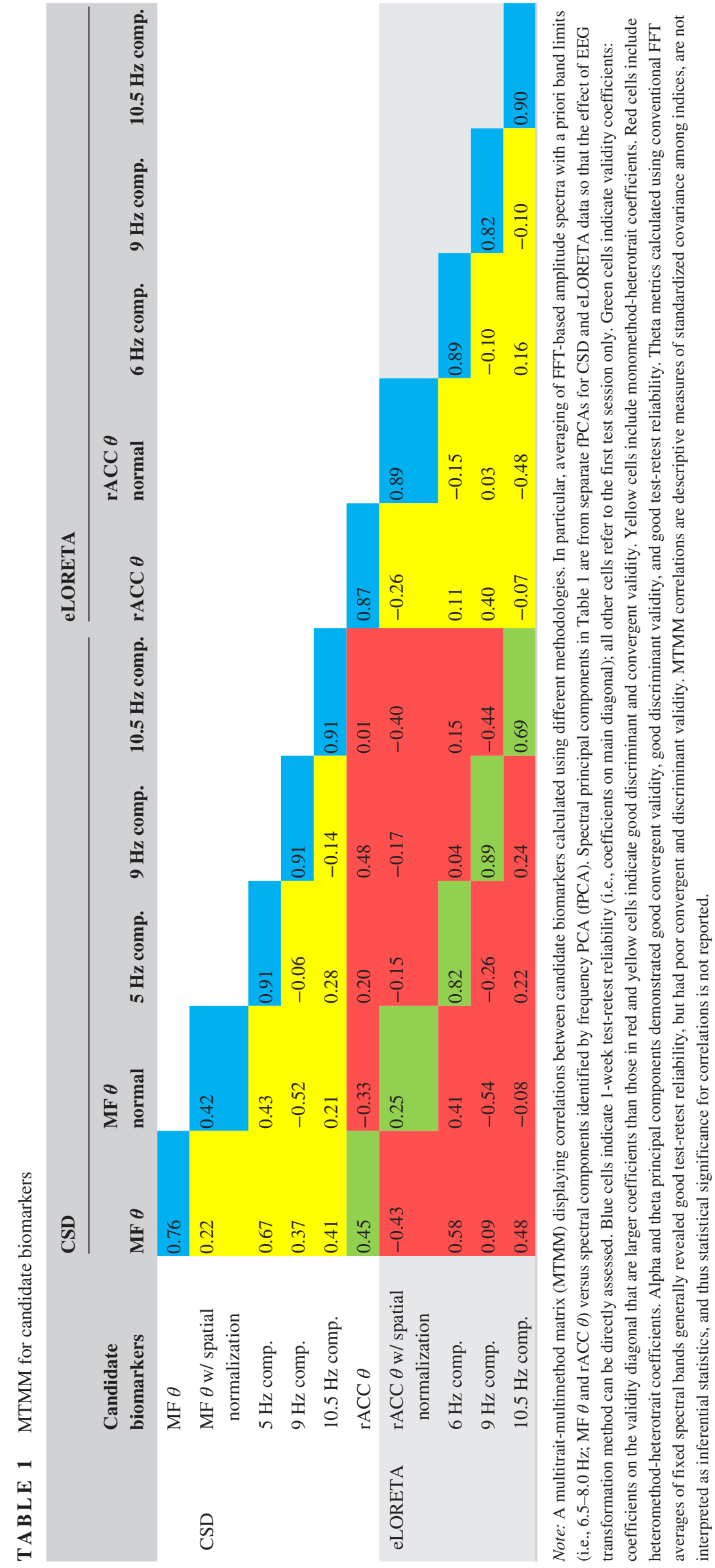


1/f amplitude. A second technique $\left(\mathrm{SNR}_{\mathrm{sg}}\right)$ calculated signal as the average cross-trial spectral amplitude and noise as the single-trial standard deviation of an amplitude spectrum. In this case, noise is assumed to be the variance across a frequency spectrum, and then standard deviations for each trial were averaged together to create an estimate of noise across the recording. For single-trial analyses, 148 random trials (i.e., all participants had at minimum 148 artifact-free trials) were selected from the first EEG recording session (74 trials each from EC and EO blocks) for calculation of $\mathrm{SNR}_{\mathrm{sgl}}$. Random selection of an equal number of epochs was used so that all participants benefited similarly from the number of trials used during averaging. Although neither SNR calculation is believed to represent "ground truth," both measure the presence of a periodic signal relative to the background EEG (noise).

To obtain estimates of internal consistency, FFT spectra from the first EEG session were averaged for theta and alpha from a random split-half of 148 epochs (i.e., 74 each, irrespective of condition).

\section{$2.4 \mid$ rACC 6.5-8 Hz current density}

Based on reports of improvement in localization accuracy with newer iterations of the LORETA source estimation package (Pascual-Marqui, 2007; Pascual-Marqui et al., 2018), we opted to analyze rACC theta using the more recent implementation. The rACC ROI was based on a rACC ROI used by Pizzagalli and others $(2001,2018)$ but varied slightly with the newer eLORETA brain. This rACC ROI included 39 voxels nearest (within $10 \mathrm{~mm}$ ) a centroid at [ $\pm 540-5$ ] in MNI (Montreal Neurological Institute) space (supporting information, Figure S3). Importantly, LORETA, sLORETA, and eLORETA versions all produced very similar estimates of rACC theta (Pearson's correlations for all pairwise comparisons between LORETA versions: for $4.5-7 \mathrm{~Hz}$, $.82 \leq r \leq .99$; for $6.5-8 \mathrm{~Hz}, .80 \leq r \leq .99$ ). Thus, eLORETA estimates were considered to be equivalent to LORETA or sLORETA estimates.

Although 4-8 Hz are typically employed as theta band limits (Chatrian et al., 1974; Kane et al., 2017), the 6.5-8 Hz band was used here for consistency with previous reports examining biomarker capability for rACC theta (e.g., Arns et al., 2015; Hunter et al., 2013; Korb et al., 2009; Pizzagalli et al., 2001). Current density was normalized (theta amplitude at each voxel/summed theta amplitude across all 6,239 voxels) and averaged across the rACC ROI. For comparison, raw theta (i.e., not normalized) was calculated as the average across rACC voxels. Rather than solely examining rACC, the current analysis also examined three additional cingulate ROIs to identify and determine the strength of other possible theta generators: dACC, sgACC (Brodmann area 25), and PCC.

\subsection{CSD-fPCA, eLORETA-fPCA, and combined CSD/eLORETA-fPCA}

Sensor-level (i.e., CSD) amplitude spectra consisting of 157 frequencies between 1 and $40 \mathrm{~Hz}(0.25 \mathrm{~Hz}$ frequency resolution) for each participant $(N=35)$, test session (2), EO/ EC condition (2), and sensor (71), resulting in a 9,940 $\times 157$ Cases $\times$ Variables data matrix, were submitted to unrestricted fPCA followed by Varimax rotation of covariance loadings (Kayser \& Tenke, 2003; Tenke \& Kayser, 2005).

The procedure used for the sensor-level CSD data was repeated for voxel-level spectral amplitude (i.e., square root of power; Tenke \& Kayser, 2005). First, the 6,239 voxels of the eLORETA brain were parcelled into 84 Brodmann areas (BAs; 42 for each hemisphere as created by the eLORETA software). This resulted in a $1,760 \times 157$ data matrix (i.e., 35 Participants $\times 2$ Conditions $\times 2$ Sessions $\times 84$ Brodmann areas and 157 Frequency bins), which was submitted to unrestricted Varimax-PCA.

We also conducted a combined CSD/eLORETAfPCA that encompassed 21,700 cases (35 Participants $\times 2$ Conditions $\times 2$ Sessions $\times[71$ Scalp sites +84 Brodmann areas]). As the separate CSD- and eLORETA-fPCAs relied on unstandardized covariance as the association matrix for component extraction (Kayser \& Tenke, 2003), and because in all likelihood CSD and eLORETA data have dissimilar covariances, each data set was scaled by their respective total covariance (i.e., the sum of the diagonal elements of the covariance matrix) so that the total covariance for each metric was equal to 1 (see Kayser \& Tenke, 2015a, for a related example of scaling covariances for this purpose). A combined CSD/eLORETA-fPCA solution was obtained because of the merits afforded by a unitary model for oscillatory phenomena often presented separately at sensor/scalp (CSD) or voxel/ brain (eLORETA) levels.

Frequency PCA followed by unrestricted Varimax rotation (Kayser \& Tenke, 2003) yields as many factors as there are linearly independent variables. In most instances, this will result in an fPCA solution comprising as many factors as there are variables (i.e., the total number of spectral frequencies), although the vast majority will explain little variance (Kayser $\&$ Tenke, 2003). For brevity and simplicity, we focused our analysis on the factors accounting for the top $99 \%$ of spectral variance, in line with previous work (Tenke \& Kayser, 2005).

All frequency PCAs were based on the average spectrum from all available trials from both recording sessions of each participant (i.e., following the procedures described in Tenke $\&$ Kayser, 2005).

\section{6 | Statistics}

Statistical analyses were conducted using native functions from the statistics toolbox of MATLAB (2018a v 9.4, The MathWorks Inc.) and custom MATLAB code. Wilcoxon signed-rank tests 
were used for examining paired-samples comparisons (i.e., condition difference between EO and EC epochs). A correction for multiple comparisons across brain regions (i.e., BAs) based on a surrogate null distribution of 1,000 random shuffles was used for evaluation of statistical significance (i.e., the so-called T-max test; Holmes, Blair, Watson, \& Ford, 1996). A corrected $p$ value $<.05$ was considered significant. Corrected $p$ values are reported unless otherwise indicated.

\section{3 | RESULTS}

\subsection{Signal quality for resting theta and alpha oscillations}

Internal consistency was excellent for both theta and alpha FFT amplitudes $(r s>.99)$. Individual spectra from
eLORETA source models, as well as SNR, are displayed in Figure 1. Individual spectral amplitudes indicated that only a few individuals were characterized by a noticeable theta peak (Figure 1b). Figure $1 \mathrm{~b}$ also shows that theta amplitude was similar for EC (top panel) and EO (bottom panel). This observation was bolstered by a robust correlation between 74 random EC and 74 random EO epochs $(r=.951)$. For $\mathrm{SNR}_{\mathrm{avg}}$, pairwise follow-up comparisons for a significant main effect of region, $F(3,102)=48.927, p<.001$, did not reveal significant differences between the rACC, sgACC, and dACC ROIs (all $p \mathrm{~s}>.57$; Figure $1 \mathrm{c}$ ). The $\mathrm{PCC}$ region demonstrated significantly less theta $\mathrm{SNR}_{\text {avg }}$ than the rACC, $t(34)=8.121$, $p<.001$. For $\mathrm{SNR}_{\mathrm{sgl}}$, repeated measures analysis of variance (ANOVA) also confirmed that $\mathrm{SNR}_{\mathrm{sgl}}$ varied across ROIs, $F(3,102)=4.700, p=.004$. Pairwise comparisons revealed that theta was weaker at the rACC than at any other ROI (all (a)

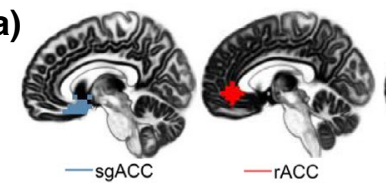

(c)
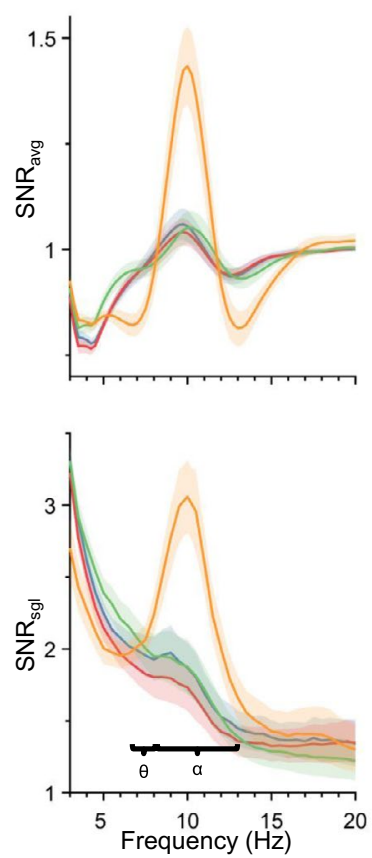
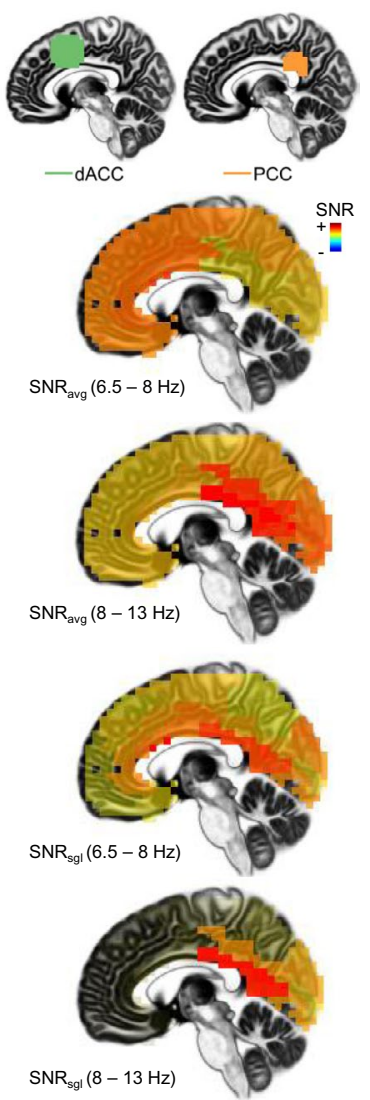

(b)
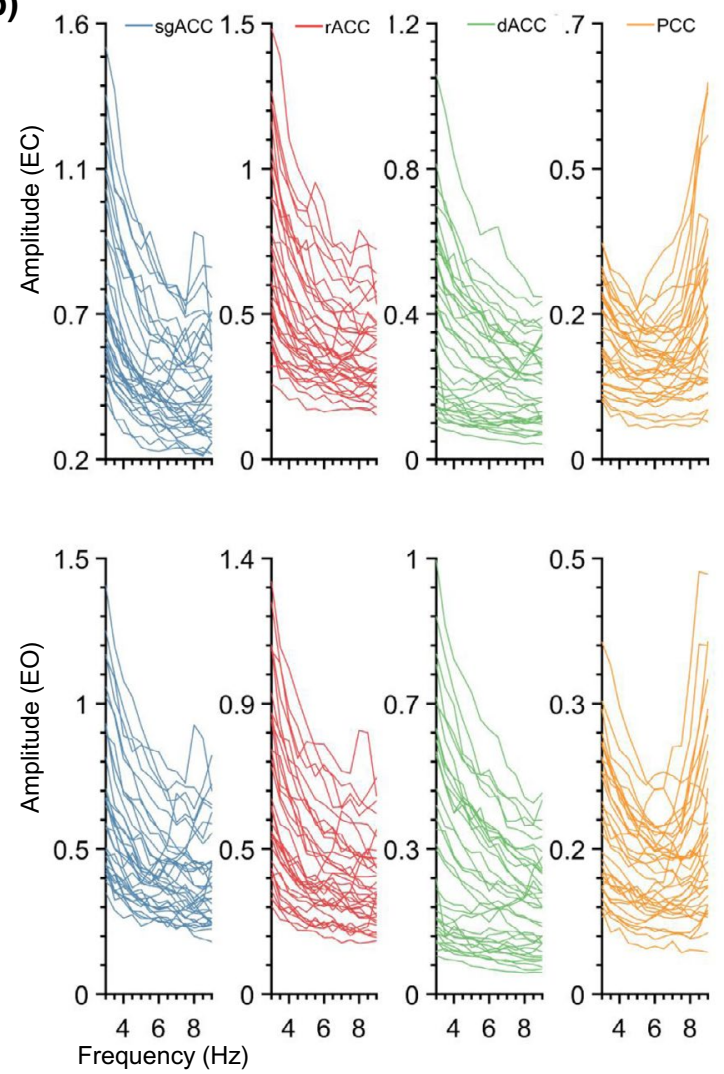
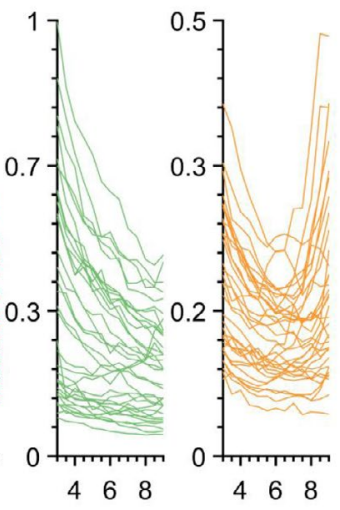

F I G U RE 1 Individual amplitude spectra and signal-to-noise ratio (SNR) for different regions of interest (ROIs) within the cingulate cortex (ACC/PCC: anterior/posterior cingulate cortex; sg: subgenual; r: rostral; d: dorsal). (a) four different ACC ROIs. From left to right: subgenual ACC (sgACC), rostral ACC (rACC), dorsal ACC (dACC), posterior cingulate cortex (PCC). (b) mean amplitude spectra for each participant (across 148 random epochs) from the first resting EEG session, displayed separately for eyes closed (EC, top) and eyes open (EO, bottom). Most individual spectra were not characterized by a distinct peak within the $4-8 \mathrm{~Hz}$ theta range. ROIs as in (a). (c) SNR operationalized as the ratio of spectral amplitude at a single frequency bin to the average amplitude of the surrounding $\pm 5 \mathrm{~Hz}$ (SNRavg, left top) and as the average cross-trial spectral amplitude in relation to the single-trial standard deviation of the amplitude spectrum (SNRsgl, right bottom; see text for computational details). Shaded regions indicate 1,000-fold bootstrapped 95\% confidence interval of the mean. In both cases, SNR was greatest for alpha-band activity at the PCC, more modest for the theta band, and theta SNR was largest for the dACC. Theta and alpha SNR is also displayed on sagittal view of eLORETA brain (right; warmer colors depict higher SNR). Theta was characterized by higher SNR at dACC and PCC regions than rACC or sgACC. Alpha was characterized by high SNR at posterior regions 
$p$ s < .007). In contrast, alpha was greatest at the PCC for both $\mathrm{SNR}_{\text {avg }}$ and $\mathrm{SNR}_{\mathrm{sgl}}($ all $p \mathrm{~s}<.001)$.

\section{2 | CSD/eLORETA-fPCA}

Factor loadings for alpha components were highly similar for CSD, eLORETA, and combined CSD/eLORETA solutions (Tucker congruence coefficient $\phi: 0.78 \geq \phi \mathrm{s} \geq 0.98$; $\phi \geq 0.85$ indicates fair similarity, and $\phi \geq 0.95$ indicates that factors are considered equal; Lorenzo-Seva \& ten Berge, 2006). The first 11 components of the combined CSD/eLORETA fPCA accounted for $99 \%$ spectral variance. Two highvariance components had peak frequencies at $8.5(4.3 \%)$ and $10.5 \mathrm{~Hz}(30.8 \%)$, and posterior topographies consistent with previous results (e.g., Tenke \& Kayser, 2005; Tenke et al., 2011; Figure S4, bottom panel). Reconstructed spectra using only these two alpha factors were then submitted to a second fPCA, which excluded frequencies above $20 \mathrm{~Hz}$ (following the procedure of Tenke et al., 2011), yielding the 9 and $10.5 \mathrm{~Hz}$ factors used for all subsequent analyses (Figure 2). Alpha component topographies and tomographies are displayed in Figure 2 separately for each resting condition (EO, $\mathrm{EC}$ ), along with their respective difference (EC - EO, or net alpha; cf. Tenke, Kayser, Abraham, Alvarenga, \& Bruder, 2015). Alpha components demonstrated the strongest alpha amplitude in parietal-occipital BAs and weak alpha amplitude in frontal regions. The EC - EO difference was maximal in parietal-occipital BAs, indicating that EC cued enhancement of alpha oscillations in parietal-occipital brain regions; however, the strength and spatial distribution of this condition effect varied with alpha component.

The $9 \mathrm{~Hz}$ component demonstrated a significant condition difference (EC - EO; Figure 3 ) in the right retrosplenial cortex and PCC (BA 30, $Z=4.59, p<.001$ ) and inferior-lateral occipital and temporal gyri (BAs 18, 29, 41; all Zs $>4.50$, all $p$ s $<.001)$.

In contrast, there were no significant condition differences for the $10.5 \mathrm{~Hz}$ component after correcting for multiple comparisons. However, when applying a more liberal statistical threshold that was used in previous work (uncorrected $p<.01$; Pizzagalli et al., 2003), several trend-level condition differences emerged for the $10.5 \mathrm{~Hz}$ component, as shown in Figure 3. These condition differences were maximal near the superior parietal lobe, right postcentral gyrus, and right fusiform gyrus (BAs 5, 3, 37; Zs $>2.50, p s<.01$ ).

While the CSD-fPCA solution revealed a distinct theta component (peak frequency $6 \mathrm{~Hz}, 1 \%$ explained variance; Figure S4) in the first $99 \%$ explained variance, this was not the case for the eLORETA- and combined CSD/eLORETAfPCA. Therefore, the search for a theta component was widened to $99.9 \%$ total variance, which revealed one lowvariance $(0.05 \%)$ factor with a peak frequency at $5 \mathrm{~Hz}$ and a midfrontal maximum (Figure 4). Factor scores for this $5 \mathrm{~Hz}$ (a)

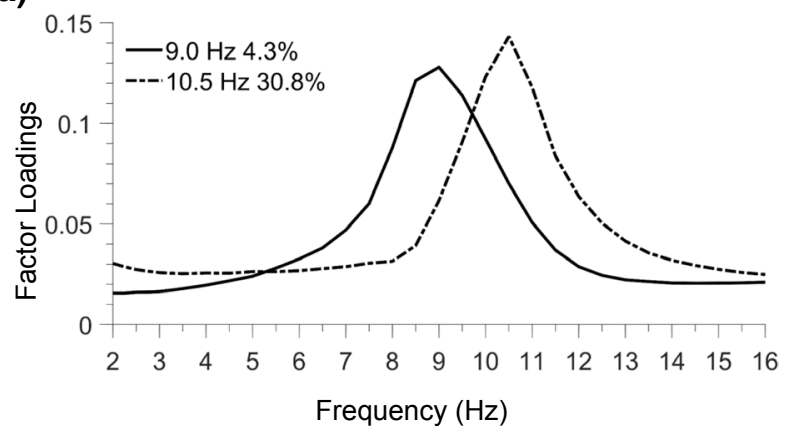

(b)

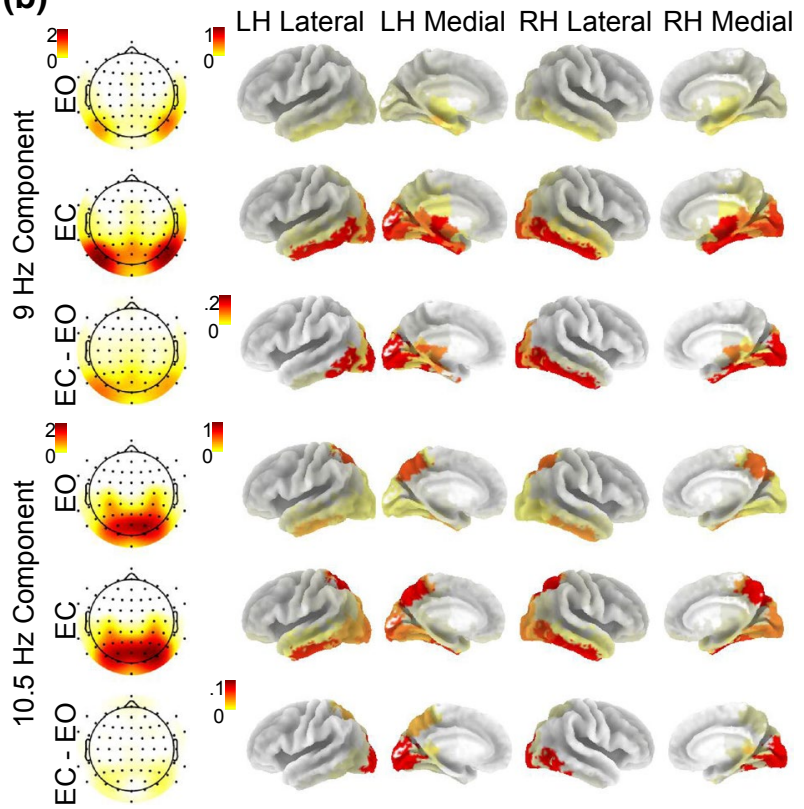

F IG URE 2 Low and high alpha components from the combined CSD/eLORETA-fPCA solution. (a) Factor loadings revealed peak frequencies at 9 and $10.5 \mathrm{~Hz}$ (low and high alpha, respectively). (b) Topographies (column 1) and tomographies (columns 2-5) of corresponding factor scores for eyes open (EO) and eyes closed (EC) conditions and for net alpha amplitude (EC minus EO) indicative of greater alpha for eyes closed, particularly for low alpha. Across CSD and ELORETA data, low alpha showed posterior-lateral maxima, whereas high alpha had a posterior-medial maximum (for full fPCA solution, see Figure $\mathrm{S} 4)$. LH = left hemisphere; $\mathrm{RH}=$ right hemisphere

principal component showed several local maxima at medial anterior regions, including premotor areas (e.g., BAs 6, 24) and the dACC. There were no significant condition differences (EC vs. EO, all $p s>.3$ ).

\section{3 | MTMM for rACC theta and posterior alpha}

Interpretation of correlations in Table 1 is based on a MTMM framework that is helpful for evaluating convergent and discriminant validity of novel psychometrics (Campbell \& Fiske, 1959). Significance tests are not a focus of the MTMM correlations-correlations are interpreted as descriptive, not 


$$
9 \mathrm{~Hz} \text { Factor }
$$$$
\text { EC - EO }
$$

(corrected $p<.05$ )
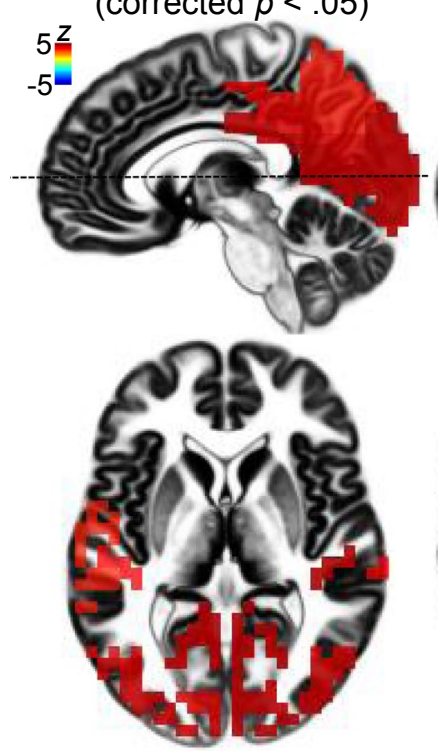

$10.5 \mathrm{~Hz}$

$\mathrm{EC}-\mathrm{EO}$

(uncorrected $p<.01$ )
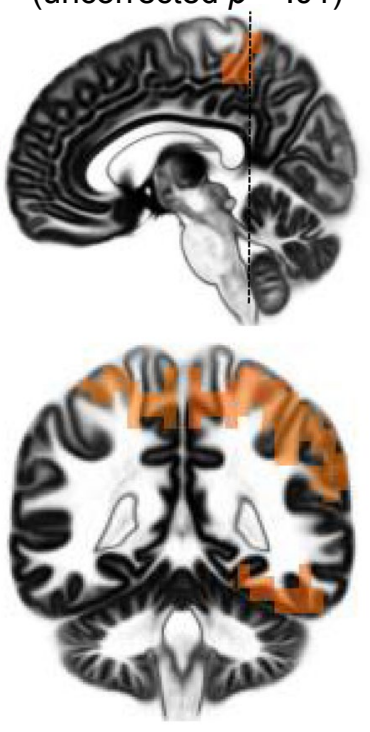

F I G URE 3 Statistical evaluation of net alpha (EC - EO) tomographies stemming from the combined CSD/eLORETA fPCA solution shown in Figure 2. Hot colors (reds and oranges) indicate greater alpha for EC than EO. Low alpha showed robust condition differences (thresholded at corrected $p<.05$ ); by comparison, condition differences for high alpha did not survive multiple comparisons correction and are presented with a more liberal threshold (uncorrected $p<.01$ ). Maximal condition differences for low alpha were near the lingual gyrus/V1, but there were also significant net low alpha effects distributed across the occipital cortex and temporal gyri. Condition differences for high alpha were maximal near the superior and inferior parietal lobe and right fusiform gyrus. Hatched lines on sagittal planes (top) indicate view for axial (bottom left) and coronal (bottom right) views

inferential—and are not reported. Table 1 displays MTMM correlations between theta and alpha metrics calculated from FFT averages using typical frequency bands and sensors or principal component scores from separate CSD or eLORETA-fPCAs. The combined CSD/eLORETA-fPCA results were not examined in the MTMM because we wanted to evaluate validity across different techniques, in this case, CSD versus ELORETA.

Within the MTMM framework, theta and alpha measures are presumed to index different neurophysiological traits (i.e., constructs) pertinent to recovery from a depressive episode. For instance, rACC theta has been linked with internetwork coordination and cognitive control (Pizzagalli, 2011), whereas posterior alpha is more often linked with arousal and vigilance (Olbrich et al., 2009; Tenke et al., 2015; Ulke et al., 2019). In contrast, CSD and eLORETA are different methods in the MTMM. The main diagonal of the MTMM (Table 1) reflects the 1-week test-retest reliability, with coefficients displayed with a blue background (test-retest reliability is
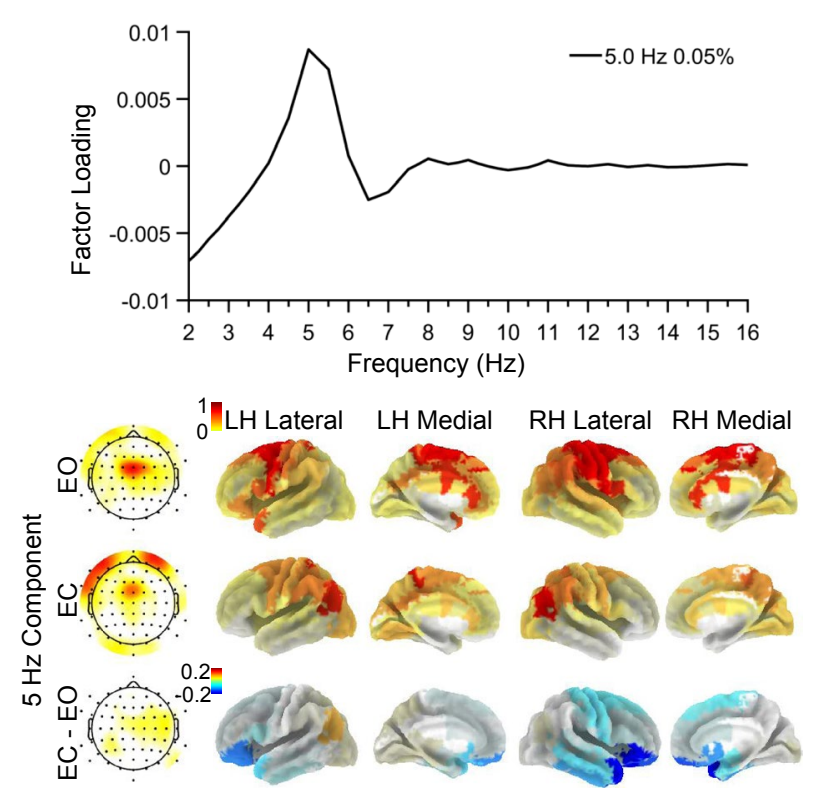

F I G URE 4 Midfrontal theta component extracted by combined CSD/eLORETA-fPCA. (top) Factor loadings revealed a peak frequency at $5 \mathrm{~Hz}$. (bottom) Overall component topography was consistent with previous work investigating midfrontal theta. Component tomography suggests sources in premotor areas, including the dACC. There were no significant condition differences (EC vs. EO) for the theta component after multiple comparisons correction (corrected $p \mathrm{~s}>.3$ ). $\mathrm{LH}=$ left hemisphere; $\mathrm{RH}=$ right hemisphere

also reported in Tenke et al., 2017, for some of these metrics). Coefficients with a green background make up the validity diagonal. Validity coefficients that are larger than coefficients with a red or yellow background suggest good convergent and discriminant validity. On one hand, the absolute magnitude of the validity coefficient speaks to convergent validity (i.e., a large correlation between traits assessed with a different measurement type). On the other hand, the relative magnitude of the validity coefficient compared to other correlations in the matrix speaks to discriminant validity. Correlations between different methods (CSD vs. eLORETA) and different traits (theta vs. alpha) are called heteromethod-heterotrait correlations, which are displayed with a red background. High correlations in the heteromethod-heterotrait triangles speak to misspecification of intertrait relationships (e.g., traits are overlapping or nonspecific), or, more generally, these correlations indicate covariance between traits and measures that would not be hypothesized to covary. Finally, the monomethod-heterotrait triangles are displayed with a yellow background. Large monomethod-heterotrait correlations indicate that a particular method contributes to significant covariance between traits. Of course, assumptions regarding what constitutes traits and methods are arguable, and MTMM interpretation is contextualized by tenability of these assumptions as well as by extant literature and theory. Nonetheless, examination of covariance patterns between candidate biomarkers can 
reveal important information about test validity beyond that inferred from bivariate correlations between predictors and outcomes (i.e., predictive validity; Campbell \& Fiske, 1959).

Theta metrics at scalp demonstrated good test-retest reliability, with the exception of spatially normalized MF $\theta$ (Table 1, blue). By comparison, intracranial theta metrics were reliable over time regardless of quantification approach. Theta component scores (i.e., via fPCA) demonstrated better convergent validity ( $r=.82$; Table 1 , green) than other theta metrics. Theta components also demonstrated high convergent and discriminant validity (i.e., larger than coefficients with yellow and red background). In contrast, whereas nonnormalized theta measures demonstrated modest convergent validity across CSD and eLORETA methods ( $r=.45)$, spatially normalized theta measures demonstrated poor convergence across transformations $(r=.25)$. Normalized and non-normalized theta metrics also demonstrated high heterotrait correlations, indicating poor discriminant validity with posterior alpha components. Moreover, spatially normalized theta demonstrated small correlations with fPCA theta components.

Posterior alpha components demonstrated good convergent validity across CSD and eLORETA fPCA decompositions (Table 1, green), good discriminant validity with theta metrics, and good test-retest reliability. For example, coefficients in validity diagonals for posterior alpha components (green background in Table 1) were larger than heteromethodheterotrait (red background) and monomethod-heterotrait (yellow background) coefficients.

Although our focus was on the qualitative comparison of MTMM coefficients, validity coefficients for frequency PCA components (theta and alpha) were significantly greater than the validity coefficients for normalized theta metrics (all $p \mathrm{~s}<.02$ ), supporting the notion that frequency PCA improves convergent validity across CSD and eLORETA transformations compared to spatial normalization.

\section{4 | DISCUSSION}

This study examined the comparative validity of two resting EEG candidate biomarkers of MDD symptom improvement in a sample of healthy adults. The present findings are in agreement with prior work noting lack of prominent theta activity during wakeful rest (Barry \& De Blasio, 2018; Cigánek, 1961; Frauscher et al., 2018; Keitel \& Gross, 2016; Schacter, 1977; Shackman et al., 2010; Tenke \& Kayser, 2005; Westmoreland \& Klass, 1986). A combination of spatial filtering, fPCA, and conventional frequency analyses indicated that theta-band activity was weak in this resting-state EEG data set from healthy adults. By comparison, posterior alpha was prominent, reliably quantified, and persistent across data transformation (CSD vs. eLORETA). Patterns of correlations between alpha and theta metrics indicated that spectral component (fPCA) measures evidenced good convergent and discriminant validity. Theta metrics computed at brain (rACC) and sensor (MF $\theta$ ) level using spatial normalization and spectral averaging of a $6.5-8 \mathrm{~Hz}$ band evidenced poor validity.

\section{1 | Findings and context: Theta}

A low-variance, $5 \mathrm{~Hz}$ theta component stemming from a combined CSD/eLORETA-fPCA solution of resting EEG was highly similar to midfrontal theta as typically described in the literature (Cavanagh \& Shackman, 2015; Schacter, 1977). This $5 \mathrm{~Hz}$ component was most prominent over frontocentral sensors and near the dACC using the eLORETA source model. Notably, this theta component accounted for a very small amount of spectral variance across participants, conditions, and scalp/brain regions. Indeed, only a few participants showed a noticeable theta peak in their mean amplitude spectra (Figure 1). These findings are consistent with the notion that theta activity is weak and infrequent during wakeful resting states (Keitel \& Gross, 2016; Vidaurre et al., 2018; Westmoreland \& Klass, 1986). In contrast, event-related and induced theta oscillations are prominent (Cavanagh \& Shackman, 2015), especially during cognitive load (Cavanagh \& Frank, 2014; Cohen, 2014a; Hsieh \& Ranganath, 2014; Li et al., 2016; Olvet \& Hajcak, 2009; Sauseng, Griesmayr, Freunberger, \& Klimesch, 2010). When theta oscillations are observed in resting EEG, oscillations tend to be brief in duration (<1 s; Vidaurre et al., 2018). This is consistent with the idea that rapid and incidental activation of theta-band processes during resting state may be masked by cross-trial averages dominated by default-mode activity, which leans toward alpha oscillations (Knyazev, Slobodskoj-Plusnin, Bocharov, \& Pylkova, 2011; Schmidt et al., 2017; Tenke \& Kayser, 2005). For example, Li and colleagues (2016) reported that frontal theta following a cognitive task predicted clinical response to antidepressant treatment, whereas baseline differences in resting frontal theta did not. It could therefore be argued that the cognitive demands studied by Li et al. (2016) likely cued theta oscillations critical for cognitive processing, which presented the researchers with measurable and pertinent variance by which to predict treatment outcome. Indeed, most work examining theta has focused on phasic theta oscillations rather than on a stable and continuous theta rhythm (Cavanagh \& Shackman, 2015; Cohen, 2014a).

Theta generators have been localized to several different regions of the brain, including the $\mathrm{rACC}$ ( $\mathrm{Li}$ et al., 2016; Pizzagalli et al., 2003; Scheeringa et al., 2008), dACC (Cohen, 2011; Debener, Ullsperger, Siegel, \& Engel, 2006; Frauscher et al., 2018; Li et al., 2016; Onton, Delorme, \& Makeig, 2005), and PCC (Martin-Buro et al., 2016). The results here indicated that, although theta peaks were apparent in the 
sgACC and rACC for some participant-level spectra, dACC had larger SNR and factor scores, suggesting that dACC may be a preferable ROI for measuring theta at rest (Arns et al., 2015; Li et al., 2016). Theta amplitude near the rACC is also prone to contamination by residual non-neurogenic artifact (Mcmenamin et al., 2010); moreover, EEG demonstrates generally poor SNR near the rACC (Goldenholz et al., 2009). These difficulties with robust measurement of a resting-state theta biomarker may also contribute to the apparent variability in theta amplitude across EMBARC research sites (Tenke et al., 2017), underscoring the difficulty of enhancing ("scaling up") theta toward reliable clinical use. Spectral component factor loadings (Figure 4), as well as visual inspection of participant spectra (Figure 1b), suggest that a 5-6 Hz peak (Cavanagh \& Shackman, 2015; Chatrian et al., 1974) may be more representative of theta than the often-used $6.5-8.0 \mathrm{~Hz}$ band. In fact, recent work examining associations between theta oscillations and MDD outcomes has adopted the more conventional 4-8 Hz theta band (Pizzagalli et al., 2018; Whitton et al., 2019). Of course, if the effectiveness of high theta $(6.5-8.0 \mathrm{~Hz})$ to predict clinical outcomes is directly related to posterior alpha components, then the alpha components are likely superior resting EEG biomarkers. Altogether, the literature suggests that assessment of theta oscillations and their underlying neural generators may be improved upon by modifying assays to include perturbation of theta circuitry (e.g., cognitive load, event-related designs; Cohen, 2014a) and with an increased focus on empirical identification and quantification of theta.

\section{2 | Findings and context: Alpha}

Posterior alpha components from an eLORETA fPCA and a combined CSD/eLORETA fPCA were highly comparable to alpha components found in previous reports examining only CSD data, both in terms of peak frequencies (i.e., about 9 and $11 \mathrm{~Hz}$ ) and band-specific topographies (lateral vs. midline occipitoparietal maxima). Source modeling yielded additional information about the differential tomographic distribution underlying these low and high alpha rhythms. Low alpha $(9 \mathrm{~Hz})$ was strongest in primary visual cortex and inferior-temporal gyri, but high alpha was strongest in anterior-superior-lateral parietal regions, especially on the right (also see Barzegaran et al., 2017, for a similar example). These eLORETA distributions are fully consistent with their CSD counterparts, but also extend previous CSD findings. For example, a deep medial source for the $9.0 \mathrm{~Hz}$ component and inferotemporal sources for both the 9.0 and $10.5 \mathrm{~Hz}$ components are only evident with the eLORETA source model. Whereas the low alpha component (peak at $9.0 \mathrm{~Hz}$ ) demonstrated a robust eyes open/closed Berger effect, the EC - EO difference for the high alpha component $(10.5 \mathrm{~Hz})$ was more modest. The strongest condition differences for low alpha were near primary visual cortex, which is consistent with a role of low alpha to inhibit ongoing activity in areas important for vision while the eyes are closed (Klimesch, 1999; Sadaghiani \& Kleinschmidt, 2016; Tenke et al., 2015). By comparison, high alpha was less affected by closing of the eyes, with trend-level condition differences apparent in parietal lobes; interestingly, these parietal high alpha sources overlap with brain regions important for cross-modal attention and working memory performance (Figure 3; Haegens, Cousijn, Wallis, Harrison, \& Nobre, 2014; Klimesch, 1999; Knyazev et al., 2011; Sadaghiani \& Kleinschmidt, 2016; Tenke et al., 2015). Moreover, it has been noted that high alpha is more likely to be affected by attention/cognition manipulations than low alpha (see Klimesch, 1999; Sadaghiani \& Kleinschmidt, 2016, for reviews). Overall, these results align with a body of work demonstrating that narrow bands of the alpha frequency are spatially and functionally dissociable (Barzegaran et al., 2017; Buzsáki, 2006; Frauscher et al., 2018; Klimesch, 1999, 2018; Sadaghiani \& Kleinschmidt, 2016).

The spectral component structure of alpha was relatively invariant to different spatial filters (CSD vs. eLORETA), including spherical splines of varying flexibility that are differentially sensitive to different generator configurations (i.e., shallow vs. deep, narrow vs. broad; Kayser \& Tenke, 2015b; Tenke \& Kayser, 2015), suggesting that the latent structure of resting alpha activity is robust to differences in identification and quantification methodology. Indeed, the latent structure of spectral data is remarkably homogeneous across studies, given that researchers have identified spectral components with 8.6 and $10.9 \mathrm{~Hz}$ peaks (Tenke \& Kayser, 2005), 9.0 and $10.5 \mathrm{~Hz}$ peaks (Tenke et al., 2011), 8.0 and $10.0 \mathrm{~Hz}$ peaks (Shackman et al., 2010), 8.5 and $10.0 \mathrm{~Hz}$ peaks (Barry \& de Blasio, 2018), and 9.0 and $10.0 \mathrm{~Hz}$ peaks (Barzegaran et al., 2017). Altogether, findings for resting EEG have been highly consistent at identifying two distinct alpha bands $(8-9 \mathrm{~Hz}$ and 10-11 Hz) using data-driven approaches like fPCA, indicating that resting-state alpha is easily quantified at rest as well as across a wide range of conditions (Tenke et al., 2015; see also Tenke et al., 2017).

\section{3 | Relationships between theta and alpha}

Interrelationships between biomarkers indicated good psychometric properties for alpha and theta spectral components (i.e., fPCA-based), whereas psychometrics for typical frontal/ rACC theta measures were poor. For example, associations between spectral measures varied sizably as a function of spatial normalization (Table 1). The correlation between raw rACC and a $9.0 \mathrm{~Hz}$ component approached the magnitude of validity coefficients, whereas normalized rACC theta was uncorrelated with the low alpha component. The opposite 
relationship was observed for the $10.5 \mathrm{~Hz}$ alpha component. Similar results were found for normalized MF $\theta$ at the scalp. Collectively, results suggest that theta metrics are influenced by different variance sources depending on whether normalization is used.

In contrast, component-based metrics demonstrated good convergent and discriminant validity. Coefficients for alpha metrics on the validity diagonal were large, indicative of convergence across measures, and distinct from the rACC theta biomarker. A similar pattern was evidenced for theta components: theta components correlated highly with one another across methods, moderately with conventional theta metrics, and demonstrated small correlations with alpha components.

As a caveat, it should be noted that power-power coupling has been observed between theta and alpha, so relationships between these measures could reflect a combination of spurious and true neural interactions (Cohen \& Van Gaal, 2013; Klimesch, 2018; Popov et al., 2018). However, spectral overlap and/or leakage seems to be a more parsimonious explanation for theta-alpha correlation during the resting state. Insofar as alpha amplitudes contribute to normalized theta amplitudes, the mere variation of using spatial normalization versus raw theta may account for discrepancies between reports (compare Arns et al., 2015 and Pizzagalli et al., 2018). In fact, some researchers have used a combination of theta and alpha activity to successfully predict MDD prognosis, for example, by averaging across a $3-12 \mathrm{~Hz}$ frequency band (Hunter et al., 2011; Leuchter, Cook, Gilmer, et al., 2009; Leuchter, Cook, Marangell, et al., 2009). Altogether, the current findings strongly indicate that posterior alpha components and a $6.5-8.0 \mathrm{~Hz}$ rACC theta metric covary, and it seems likely that suboptimal measurement of rACC theta contributes to an undesirable conflation of frontal theta and posterior alpha biomarkers.

\section{4 | Limitations}

While this study employed both design and analysis pipelines of previous biomarker research to improve comparability (Tenke et al., 2017), conventional cross-trial averaging of the resting state is a poor representation of true dynamic internetwork communication (Allen \& Cohen, 2010; Buzsáki, 2006; Vidaurre et al., 2018). Accordingly, the study is limited by a lack of parallel event-related data for examining theta and theta-alpha interactions, analyses amenable to aperiodic signals, and a direct comparison of EEG oscillations during task performance versus resting. Recordings of event-related EEG may have also facilitated interpretation of functional differences in posterior alpha components, for instance, by linking different alpha components to visual processing and/ or working memory capacity (e.g., Haegens et al., 2014; Tenke et al., 2015).
Although fPCA has the advantage of providing a simple and straightforward linear representation of the latent variance structure within a given data set, it has the disadvantage that it may not "carve nature at its joints." Specifically, Varimax-fPCA, as used here, is a linear decomposition that reveals orthogonal spectral components (e.g., Tenke \& Kayser, 2005), although it is probable that neural activity is neither linear nor orthogonal (e.g., Delorme et al., 2012). Whereas an orthogonal rotation provides the advantage of maintaining components that do not share variance and are in this sense parsimonious (Kayser \& Tenke, 2005, 2006a), oblique rotation methods may provide superior estimates under certain conditions (Barry \& De Blasio, 2018; Dien, Beal, \& Berg, 2005; Scharf \& Nestler, 2018). Recent work with relaxed orthogonality constraints has demonstrated a similar component structure to the present findings, albeit with an overall improved component loading morphology (e.g., non-negative component loadings; Barry \& De Blasio, 2018). These methodological issues will require further study in the context of spectral decomposition. Nonetheless, the simplified representation afforded by the present approach is unique, replicable, and consistent with prior work (Tenke \& Kayser, 2005; Tenke et al., 2011) and therefore adequate to address the questions posed in this report.

Notwithstanding that the present findings firmly convey the notion that a combination of weak rACC theta, spectral leakage, and spatial normalization are a parsimonious explanation for variable findings across studies, it is important to note that these studies also differed in several other critical aspects. First, the present data were from healthy adults only and did not include resting EEG from MDD patients, which have been characterized by larger theta amplitudes at rest (Arns et al., 2015). Arguably, a more valid and robust measure of theta should be a better predictor of clinical outcomes; however, this was not directly tested in this report and should therefore be a focus in future work examining comparative predictive validity for treatment outcome in MDD patients (Trivedi et al., 2016). Second, previous treatment failure with antidepressant medication (Hunter et al., 2013), differences in MDD severity (compare Arns et al., 2015 to Pizzagalli et al., 2018), use of relative frequency indices (e.g., Leuchter et al., 2017), and variation in time between EEG recording and treatment initiation (Hunter et al., 2013) constitute additional moderators (see Pizzagalli et al., 2018, for examples) of the relationship between theta and symptom improvement in MDD. Future work will need to examine how these putative moderators affect prediction of clinical outcomes in individuals with MDD.

\section{5 | Toward improving theta quantification}

The present findings argue for several methodological improvements to theta quantification. First, researchers should 
consider adopting reference-free (Kayser \& Tenke, 2010, 2015b, 2015c; Tenke \& Kayser, 2012; Nunez et al., 2019) and multivariate data-decomposition techniques (Bridwell et al., 2018; Delorme \& Makeig, 2004; Tenke \& Kayser, 2005) as a general strategy for analyzing EEG data. The present findings showing enhanced validity for CSD/eLORETAfPCA spectral components attest to the importance and benefits of this strategy. Second, conventional spectral averaging of rACC theta should pivot toward event-related and induced designs rather than relying on a resting-state paradigm. Third, examining resting-state theta may significantly benefit from approaches that oversample theta bursts or are otherwise sensitive to aperiodic theta oscillations. We describe these possible improvements and their rationale in more detail below.

The quantification of observations (neural, self-report, or otherwise) into latent variables that reflect unobservable constructs of interest is of considerable interest and importance to the field (e.g., Meehl, 1995; Patrick \& Hajcak, 2016). EEG source separation techniques facilitate interpretation, identification, and quantification of EEG signals by reducing observed EEG variance into latent variables (e.g., components) that index some unobserved neural generators (Barry \& De Blasio, 2018; Cohen, 2017a, 2017b; Delorme et al., 2012; Kayser \& Tenke, 2010; Tenke \& Kayser, 2005). This is important because signal mixing in the raw EEG dilutes measurement of variance pertinent to psychological constructs of interest. Spatial normalization should be avoided in this regard because it enhances signal mixing. At the very least, results from spatially normalized and raw data should be reported in parallel. Source separation techniques also facilitate localization of neural activity to specific brain regions and produce results that are invariant to EEG reference choice (see Cohen, 2017a; Delorme \& Makeig, 2004; Kayser \& Tenke, 2010, 2015b; Pascual-Marqui, Michel, \& Lehmann, 1994, for examples and detailed discussions of this persistent pitfall). This is important for clinical utility, as specific brain regions likely have differential relatedness to psychiatric outcomes, and EEG reference choices will vary from clinic to clinic. There are myriad techniques and tools available to accomplish these aims, yet it remains to be seen which specific techniques are best for clinical prediction (Bridwell et al., 2018; Delorme et al., 2012; Jaworska et al., 2019). Nonetheless, pivoting toward analyses focused on latent variables should substantially advance biomarker development and utility.

Task-based theta is likely to be a more robust and valid measure of cognitive control than resting theta (e.g., Li et al., 2016; Schacter, 1977), especially when using multisecond FFT averages. Resting theta is confounded by sleepiness and ocular artifact (Schacter, 1977; Strijkstra et al., 2003), resting theta oscillations are more likely to appear as transient bursts than a continuous rhythm (e.g., Keitel \& Gross,
2016; Vidaurre et al., 2018), and resting theta is inversely correlated with default mode activity (Scheeringa et al., 2008). In this way, conventional FFT-based analyses computed over multiple seconds of resting EEG are poorly suited for assessing random and aperiodic theta bursts. By comparison, when theta bursts are presumed to be more frequent and sustained, FFT may be suitable as is the case when theta activity is induced by sustained cognitive load (e.g., during a memory encoding period or during mental computation; $\mathrm{Li}$ et al., 2016; Schacter, 1977). Similarly, event-related designs benefit from time-locked theta bursts that consistently appear over trials, whereas the influence of background EEG is attenuated by averaging. Thus, clinical neuroscience could refocus its efforts toward induced and event-related theta when applying conventional FFT analyses for quantification of frontal theta.

Alternatively, FFT analyses may be amenable to assessing resting theta when theta bursts are oversampled prior to averaging. For example, researchers can comb through the resting state for specific neural events (e.g., oscillatory bursts) and then oversample these events for averaging. Specifically, Allen and Cohen (2011) extracted the top 1\% of alpha bursts from the resting EEG as their indicator of alpha activity and found that the magnitude of the top $1 \%$ of alpha bursts accounted for a remarkable $42 \%$ of the variance in prediction of depression status (history or current depression). A similar approach could also be adopted with rACC theta: researchers can apply a narrow-band temporal filter to continuous EEG for the theta band (e.g., 4-8 Hz) and identify time points from the continuous EEG corresponding to large theta bursts (at FCz, for example), and then analyze only epochs with large theta bursts using a typical FFT to calculate spectral amplitude. Another approach by Cohen (2017b) created epochs time locked to oscillatory bursts and then submitted these epochs to an EEG source separation technique (leaving out a substantial amount of the remaining background EEG). This approach guides source separation based upon experimenter theory and/or interest (i.e., the specific neural events that were oversampled); moreover, Cohen (2017b) demonstrated convincingly that this technique improves SNR, mitigates multiple comparisons, and simplifies EEG analyses (also see Parra \& Sajda, 2003; Parra, Spence, Gerson, \& Sajda, 2005). These results underscore the importance of transient neural events buried in the continuous EEG that are typically weakened by a conventional FFT approach that relies on averaging across all resting epochs.

Notwithstanding that this report did not directly investigate all of these considerations, known limitations of FFT spectral averaging, along with findings from previous studies, strongly suggests that implementing techniques that circumvent these pitfalls of conventional spectral analyses should improve the clinical utility of resting-state EEG dynamics. 


\subsection{Conclusion}

In close agreement with prior research, two distinct posterior alpha components dominated the resting EEG spectrum. Most importantly, both posterior alpha components revealed psychometric qualities supportive of their continued development as candidate EEG biomarkers: good reliability across sessions, convergent validity between methods (CSD and eLORETA), and discriminant validity with rACC theta. In contrast, rACC theta showed relatively low convergent validity across methods and poor discriminant validity with posterior alpha components. Nonetheless, a low-variance theta component demonstrated substantial advantages over rACC theta as is typically calculated, especially with regard to convergent and discriminant validity. Overall, these findings argue against the continued use of the rACC theta metric as originally proposed (i.e., recorded at rest, spatial normalization, fixed band limits). Instead, task-based assays, empirical identification of meaningful theta sources, and analyses that are amenable to aperiodic theta dynamics may be a more promising avenue for identifying theta biomarkers with high clinical utility (Iosifescu, 2011; Stewart, Coan, Towers, \& Allen, 2011; Tenke et al., 2011; Wade \& Iosifescu, 2016; Widge et al., 2018).

\section{ACKNOWLEDGMENTS}

This research was funded by National Institute of Mental Health (NIMH) award MH115299 (JK). EEG data were obtained as part of the EMBARC study under NIMH awards MH092221 (MHT) and MH092250 (McGrath, P. J.; Parsey, R. V.; MMW). We greatly appreciate helpful comments from Tarik Bel-Bahar on an earlier version of this manuscript.

\section{CONFLICT OF INTEREST}

In the last 3 years, the authors report the following financial disclosures for activities unrelated to the current research: JK: funding from NIMH and the Templeton Foundation; DAP: funding from NIMH, Brain and Behavior Research Foundation, and the Dana Foundation; consulting fees from Akili Interactive Labs, BlackThorn Therapeutics, Boehreinger Ingelheim, CompassPathway, Posit Science, and Takeda Pharmaceuticals USA and an honorarium from Alkermes for activities unrelated to the current study; MHT (lifetime disclosures): research support from the Agency for Healthcare Research and Quality, Cyberonics Inc., National Alliance for Research in Schizophrenia and Depression, National Institute of Mental Health, National Institute on Drug Abuse, National Institute of Diabetes and Digestive and Kidney Diseases, Johnson \& Johnson, and consulting and speaker fees from Abbott Laboratories Inc., Akzo (Organon Pharmaceuticals Inc.), Allergan Sales LLC, Alkermes, AstraZeneca, Axon
Advisors, Brintellix, Bristol-Myers Squibb Company, Cephalon Inc., Cerecor, Eli Lilly \& Company, Evotec, Fabre Kramer Pharmaceuticals Inc., Forest Pharmaceuticals, GlaxoSmithKline, Health Research Associates, Johnson \& Johnson, Lundbeck, MedAvante Medscape, Medtronic, Merck, Mitsubishi Tanabe Pharma Development America Inc., MSI Methylation Sciences Inc., Nestle Health Science-PamLab Inc., Naurex, Neuronetics, One Carbon Therapeutics Ltd., Otsuka Pharmaceuticals, Pamlab, Parke-Davis Pharmaceuticals Inc., Pfizer Inc., PgxHealth, Phoenix Marketing Solutions, Rexahn Pharmaceuticals, Ridge Diagnostics, Roche Products Ltd., Sepracor, SHIRE Development, Sierra, SK Life and Science, Sunovion, Takeda, Tal Medical/Puretech Venture, Targacept, Transcept, VantagePoint, Vivus, and Wyeth-Ayerst Laboratories; MMW: funding from NIMH, the National Alliance for Research on Schizophrenia and Depression (NARSAD), the Sackler Foundation, and the Templeton Foundation; royalties from the Oxford University Press, Perseus Press, the American Psychiatric Association Press, and MultiHealth Systems. All other authors report no biomedical financial interests or potential conflicts of interest.

\section{ORCID}

Ezra E. Smith (D) https://orcid.org/0000-0001-5163-7827

Jürgen Kayser (iD https://orcid.org/0000-0001-5309-1573

\section{REFERENCES}

Allen, J. J. B., \& Cohen, M. X. (2010). Deconstructing the "resting" state: Exploring the temporal dynamics of frontal alpha asymmetry as an endophenotype for depression. Frontiers in Human Neuroscience, 4, 232. https://doi.org/10.3389/fnhum.2010.00232

Alschuler, D. M., Tenke, C. E., Bruder, G. E., \& Kayser, J. (2014). Identifying electrode bridging from electrical distance distributions: A survey of publicly-available EEG data using a new method. Clinical Neurophysiology, 125, 484-490. https://doi.org/10.1016/j. clinph.2013.08.024

Arns, M., Bruder, G., Hegerl, U., Spooner, C., Palmer, D. M., Etkin, A., ... Gordon, E. (2016). EEG alpha asymmetry as a gender-specific predictor of outcome to acute treatment with different antidepressant medications in the randomized iSPOT-D study. Clinical Neurophysiology, 127(1), 509-519. https://doi.org/10.1016/j. clinph.2015.05.032

Arns, M., Etkin, A., Hegerl, U., Williams, L. M., DeBattista, C., Palmer, D. M., ... Gordon, E. (2015). Frontal and rostral anterior cingulate (rACC) theta EEG in depression: Implications for treatment outcome? European Neuropsychopharmacology, 25(8), 1190-1200. https://doi.org/10.1016/j.euroneuro.2015.03.007

Arns, M., Gordon, E., \& Boutros, N. N. (2017). EEG Abnormalities Are Associated With Poorer Depressive Symptom Outcomes With Escitalopram and Venlafaxine-XR, but Not Sertraline: Results From the Multicenter Randomized iSPOT-D Study. Clinical EEG and Neuroscience, 48(1), 33-40. https://doi.org/10.1177/1550059415 621435 
Aurlien, H., Gjerde, I. O., Aarseth, J. H., Eldøen, G., Karlsen, B., Skeidsvoll, H., \& Gilhus, N. E. (2004). EEG background activity described by a large computerized database. Clinical Neurophysiology, 115(3), 665-673. https://doi.org/10.1016/j.clinph.2003.10.019

Barry, R. J., \& De Blasio, F. M. (2018). EEG frequency PCA in EEGERP dynamics. Psychophysiology, 55(5), https://doi.org/10.1111/ psyp. 13042

Barzegaran, E., Vildavski, V. Y., \& Knyazeva, M. G. (2017). Fine structure of posterior alpha rhythm in human EEG: Frequency components, their cortical sources, and temporal behavior. Scientific Reports, 7(1), 1-12. https://doi.org/10.1038/s41598-017-08421-z

Baskaran, A., Farzan, F., Milev, R., Brenner, C. A., Alturi, S., Pat McAndrews, M., ... Kennedy, S. H. (2017). The comparative effectiveness of electroencephalographic indices in predicting response to escitalopram therapy in depression: A pilot study. Journal of Affective Disorders, 227, 542-549. https://doi.org/10.1016/j.jad.2017.10.028

Bridwell, D. A., Cavanagh, J. F., Collins, A. G. E., Nunez, M. D., Srinivasan, R., Stober, S., \& Calhoun, V. D. (2018). Moving beyond ERP components: A selective review of approaches to integrate EEG and behavior. Frontiers in Human Neuroscience, 12, 1-17. https://doi.org/10.3389/fnhum.2018.00106

Broadway, J. M., Holtzheimer, P. E., Hilimire, M. R., Parks, N. A., Devylder, J. E., Mayberg, H. S., \& Corballis, P. M. (2012). Frontal theta cordance predicts 6-month antidepressant response to subcallosal cingulate deep brain stimulation for treatment-resistant depression: A pilot study. Neuropsychopharmacology, 37(7), 1764-1772. https://doi.org/10.1038/npp.2012.23

Bruder, G. E., Sedoruk, J. P., Stewart, J. W., McGrath, P. J., Quitkin, F. M., \& Tenke, C. E. (2008). Electroencephalographic alpha measures predict therapeutic response to a selective serotonin reuptake inhibitor antidepressant: Pre- and post-treatment findings. Biological Psychiatry, 63(12), 1171-1177. https://doi.org/10.1016/j.biops ych.2007.10.009

Bruder, G. E., Tenke, C. E., Kayser, J. (2013). Electrophysiological predictors of clinical response to antidepressants. In J. J. Mann, P. J. McGrath, and S. P. Roose (Eds.), The Clinical Handbook for the Management of Mood Disorders (pp. 380-393). New York: Cambridge University Press. ISBN: 978-1-107-02463-2. https://doi. org/10.1017/cbo9781139175869

Buzsáki, G. (2006). Rhythms of the brain. New York, NY, US: Oxford University Press. http://dx.doi.org/10.1093/acprof:oso/9780195301 069.001.0001

Campbell, D. T., \& Fiske, D. W. (1959). Convergent and discriminant validation by the multitrait-multimethod matrix. Psychological Bulletin, 56(2), 88-105. https://doi.org/10.1037/h0046016

Cavanagh, J. F., \& Frank, M. J. (2014). Frontal theta as a mechanism for cognitive control. Trends in Cognitive Sciences, 18(8), 414-421. https://doi.org/10.1016/j.tics.2014.04.012

Cavanagh, J. F., \& Shackman, A. J. (2015). Frontal midline theta reflects anxiety and cognitive control: Meta-analytic evidence. Journal of Physiology: Paris, 109, 3-15. https://doi.org/10.1016/j. jphysparis.2014.04.003

Chatrian, G. E., Bergamini, L., Dondey, M., Klass, D. W., LennoxBuchthal, M., \& Petersen, I. (1974). A glossary of terms most commonly used by clinical electroencephalographers. Electroencephalography and Clinical Neurophysiology, 37(5), 538548. https://doi.org/10.1016/0013-4694(74)90099-6

Chiang, A. K. I., Rennie, C. J., Robinson, P. A., van Albada, S. J., \& Kerr, C. C. (2011). Age trends and sex differences of alpha rhythms including split alpha peaks. Clinical Neurophysiology, 122(8), 1505-1517. https://doi.org/10.1016/j.clinph.2011.01.040

Cigánek, L. (1961). Theta-discharges in the middle-line-EEG symptom of temporal lobe epilepsy. Electroencephalography and Clinical Neurophysiology, 13(5), 669-673. https://doi. org/10.1016/0013-4694(61)90099-2

Cohen, M. X. (2011). Error-related medial frontal theta activity predicts cingulate-related structural connectivity. NeuroImage, 55(3), 1373-1383. https://doi.org/10.1016/j.neuroimage.2010.12.072

Cohen, M. X. (2014a). A neural microcircuit for cognitive conflict detection and signaling. Trends in Neurosciences, 37(9), 480-490. https://doi.org/10.1016/j.tins.2014.06.004

Cohen, M. X. (2014b). Analyzing neural time series data: Theory and practice. Cambridge, MA: MIT Press.

Cohen, M. X. (2017a). Comparison of linear spatial filters for identifying oscillatory activity in multichannel data. Journal of Neuroscience Methods, 278, 1-12. https://doi.org/10.1016/j.jneum eth.2016.12.016

Cohen, M. X. (2017b). Multivariate cross-frequency coupling via generalized eigendecomposition. Elife, 6, 1-26. https://doi.org/10.7554/ elife. 21792

Cohen, M. X., \& Gulbinaite, R. (2017). Rhythmic entrainment source separation: Optimizing analyses of neural responses to rhythmic sensory stimulation. NeuroImage, 147, 43-56. https://doi. org/10.1016/j.neuroimage.2016.11.036

Cohen, M. X., \& van Gaal, S. (2013). Dynamic interactions between large-scale brain networks predict behavioral adaptation after perceptual errors. Cerebral Cortex, 23(5), 1061-1072. https://doi. org/10.1093/cercor/bhs069

Cohen, M. X., \& van Gaal, S. (2014). Subthreshold muscle twitches dissociate oscillatory neural signatures of conflicts from errors. NeuroImage, 86, 503-513. https://doi.org/10.1016/j.neuro image.2013.10.033

Debener, S., Ullsperger, M., Siegel, M., \& Engel, A. K. (2006). Singletrial EEG-fMRI reveals the dynamics of cognitive function. Trends in Cognitive Sciences, 10(12), 558-563. https://doi.org/10.1016/ j.tics.2006.09.010

Delorme, A., \& Makeig, S. (2004). EEGLAB: An open source toolbox for analysis of single-trial EEG dynamics including independent component analysis. Journal of Neuroscience Methods, 134, 9-21. https://doi.org/10.1016/j.jneumeth.2003.10.009

Delorme, A., Palmer, J., Onton, J., Oostenveld, R., \& Makeig, S. (2012). Independent EEG sources are dipolar. PLoS ONE, 7(2), e30135. https://doi.org/10.1371/journal.pone.0030135

Dien, J., Beal, D. J., \& Berg, P. (2005). Optimizing principal components analysis of event-related potentials: Matrix type, factor loading weighting, extraction, and rotations. Clinical Neurophysiology, 116, 1808-1825. https://doi.org/10.1016/j.clinph.2004.11.025

First, M. B., Gibbon, M., Spitzer, R. L., \& Williams, J. B. (1996). User's guide for the structured clinical interview for DSM-IV axis I Disorders-Research version. New York, NY: Biometrics Research Department, New York State Psychiatric Institute.

Frauscher, B., von Ellenrieder, N., Zelmann, R., Doležalová, I., Minotti, L., Olivier, A., ... Gotman, J. (2018). Atlas of the normal intracranial electroencephalogram: Neurophysiological awake activity in different cortical areas. Brain, 141(4), 1130-1144. https://doi. org/10.1093/brain/awy035

Goldenholz, D. M., Ahlfors, S. P., Ha, M. S., Sharon, D., Ishitobi, M., Vaina, L. M., \& Stufflebeam, S. M. (2009). Mapping the 
signal-to-noise-ratios of cortical sources in magnetoencephalography and electroencephalography. Human Brain Mapping, 1086(4), 1077-1086. https://doi.org/10.1002/hbm.20571

Haegens, S., Cousijn, H., Wallis, G., Harrison, P. J., \& Nobre, A. C. (2014). Inter- and intra-individual variability in alpha peak frequency. NeuroImage, 92, 46-55. https://doi.org/10.1016/j.neuro image.2014.01.049

Holmes, A. P., Blair, R. C., Watson, H. J. D. G., \& Ford, I. (1996). Nonparametric analysis of statistic images from functional mapping experiments. Journal of Cerebral Blood Flow and Metabolism, 16(1), 7-22. https://doi.org/10.1097/00004647199601000-00002

Hsieh, L., \& Ranganath, C. (2014). Frontal midline theta oscillations during working memory maintenance and episodic encoding and retrieval. NeuroImage, 85, 721-729. https://doi.org/10.1016/j.neuro image.2013.08.003

Hunter, A. M., Cook, I. A., Greenwald, S., Tran, M. L., Miyamoto, K. N., Leuchter, A. F., ... Mcmahon, L. L. (2011). The Antidepressant Treatment Response (ATR) index and treatment outcomes in a placebo-controlled trial of fluoxetine. Journal of Clinical Neurophysiology, 28(5), 478-482. https://doi.org/10.1016/j.psyne uen.2009.06.003

Hunter, A. M., Korb, A. S., Cook, I. A., \& Leuchter, A. F. (2013). Rostral anterior cingulate activity in major depressive disorder: State or trait marker of responsiveness to medication? The Journal of Neuropsychiatry and Clinical Neurosciences, 25(2), 126-133. https://doi.org/10.1176/appi.neuropsych.11110330

Iosifescu, D. V. (2011). Electroencephalography-derived biomarkers of antidepressant response. Harvard Review of Psychiatry, 19(3), 144-154. https://doi.org/10.3109/10673229.2011.586549

Iosifescu, D. V., Greenwald, S., Devlin, P., Mischoulon, D., Denninger, J. W., Alpert, J. E., \& Fava, M. (2009). Frontal EEG predictors of treatment outcome in major depressive disorder. European Neuropsychopharmacology, 19(11), 772-777. https://doi. org/10.1016/j.euroneuro.2009.06.001

Jaworska, N., de la Salle, S., Ibrahim, M.-H., Blier, P., \& Knott, V. (2019). Leveraging machine learning approaches for predicting antidepressant treatment response using electroencephalography (EEG) and clinical data. Frontiers in Psychiatry, 9, 1-16. https:// doi.org/10.3389/fpsyt.2018.00768

Kandilarova, S. S., Terziyski, K. V., Draganova, A. I., Stoyanov, D. S., Akabaliev, V. H., \& Kostianev, S. S. (2017). Response to pharmacological treatment in major depression predicted by electroencephalographic alpha power-A pilot naturalistic study. Folia Medica, 59(3), 318-325. https://doi.org/10.1515/ folmed-2017-0040

Kane, N., Acharya, J., Beniczky, S., Caboclo, L., Finnigan, S., Kaplan, P. W., ... van Putten, M. J. A. M. (2017). A revised glossary of terms most commonly used by clinical electroencephalographers and updated proposal for the report format of the EEG findings. Revision 2017. Clinical Neurophysiology Practice, 2, 170-185. https://doi. org/10.1016/j.cnp.2017.07.002

Kayser, J. (2009). Current source density (CSD) interpolation using spherical splines-CSD Toolbox (Version 1.1). Retrieved from http://psychophysiology.cpmc.columbia.edu/Software/CSDtoolbox

Kayser, J., \& Tenke, C. E. (2003). Optimizing PCA methodology for ERP component identification and measurement: theoretical rationale and empirical evaluation. Clinical Neurophysiology, 114(12), 2307-2325. https://doi.org/10.1016/s1388-2457(03)00241-4
Kayser, J., \& Tenke, C. E. (2005). Trusting in or breaking with convention: Towards a renaissance of principal components analysis in electrophysiology. Clinical Neurophysiology, 116(8), 1747-1753. https://doi.org/10.1016/j.clinph.2005.03.020

Kayser, J., \& Tenke, C. E. (2006a). Consensus on PCA for ERP data, and sensibility of unrestricted solutions. Clinical Neurophysiology, 117(3), 703-707. https://doi.org/10.1016/j.clinph.2005.11.015

Kayser, J., \& Tenke, C. E. (2006b). Principal components analysis of Laplacian waveforms as a generic method for identifying ERP generator patterns: I. Evaluation with auditory oddball tasks. Clinical Neurophysiology, 117(2), 348-368. https://doi.org/10.1016/ j.clinph.2005.08.034

Kayser, J., \& Tenke, C. E. (2006c). Electrical distance as a referencefree measure for identifying artifacts in multichannel electroencephalogram (EEG) recordings. Psychophysiology, 43, S51. Retrieved from http://psychophysiology.cpmc.columbia.edu/mmedia/SPR20 06/ElecDistArti.pdf

Kayser, J., \& Tenke, C. E. (2010). In search of the Rosetta Stone for scalp EEG: Converging on reference-free techniques. Clinical Neurophysiology, 121(12), 1973-1975. https://doi.org/10.1016/ j.clinph.2010.04.030

Kayser, J., \& Tenke, C. E. (2015a). Hemifield-dependent N1 and eventrelated theta/delta oscillations: An unbiased comparison of surface Laplacian and common EEG reference choices. International Journal of Psychophysiology, 97(3), 258-270. https://doi.org/ 10.1016/j.ijpsycho.2014.12.011

Kayser, J., \& Tenke, C. E. (2015b). Issues and considerations for using the scalp surface Laplacian in EEG/ERP research: A tutorial review. International Journal of Psychophysiology, 97(3), 189-209. https:// doi.org/10.1016/j.ijpsycho.2015.04.012

Kayser, J., \& Tenke, C. E. (2015c). On the benefits of using surface Laplacian (current source density) methodology in electrophysiology. International Journal of Psychophysiology, 97(3), 171-173. https://doi.org/10.1016/j.ijpsycho.2015.06.001

Kayser, J., Tenke, C. E., \& Debener, S. (2000). Principal components analysis (PCA) as a tool for identifying EEG frequency bands: I. Methodological considerations and preliminary findings. Psychophysiology, 37, S54. Retrieved from http://psychophysiology. cpmc.columbia.edu/mmedia/SPR2000/fPCA1.pdf

Keitel, A., \& Gross, J. (2016). Individual human brain areas can be identified from their characteristic spectral activation fingerprints. PLoS Biology, 14(6), 1-22. https://doi.org/10.1371/journal.pbio.1002498

Klimesch, W. (1999). EEG alpha and theta oscillations reflect cognitive and memory performance: A review and analysis. Brain Research Reviews, 29(2-3), 169-195. https://doi.org/10.1016/ S0165-0173(98)00056-3

Klimesch, W. (2018). The frequency architecture of brain and brainbody oscillations: An analysis. European Journal of Neuroscience, 48(7), 2431-2453. https://doi.org/10.1111/ejn.14192

Knott, V., Mahoney, C., Kennedy, S., \& Evans, K. (2000). Pre-treatment EEG and it's relationship to depression severity and paroxetine treatment outcome. Pharmacopsychiatry, 33(6), 201-205. https://doi. org/10.1055/s-2000-8356

Knott, V. J., Telner, J. I., Lapierre, Y. D., Browne, M., \& Horn, E. R. (1996). Quantitative EEG in the prediction of antidepressant response to imipramine. Journal of Affective Disorders, 39(3), 175184. https://doi.org/10.1016/0165-0327(96)00003-1

Knyazev, G. G., Slobodskoj-Plusnin, J. Y., Bocharov, A. V., \& Pylkova, L. V. (2011). The default mode network and EEG alpha oscillations: 
An independent component analysis. Brain Research, 1402, 67-79. https://doi.org/10.1016/j.brainres.2011.05.052

Korb, A. S., Hunter, A. M., Cook, I. A., \& Leuchter, A. F. (2009). Rostral anterior cingulate cortex theta current density and response to antidepressants and placebo in major depression. Clinical Neurophysiology, 120(7), 1313-1319. https://doi.org/10.1016/ j.jeconom.2010.01.006

Kubicki, S., Herrmann, W. M., Fichte, K., \& Freund, G. (1979). Reflections on the topics: EEG frequency bands and regulation of vigilance. Pharmacopsychiatry, 12(2), 237-245. https://doi.org/ 10.1055/s-0028-1094615

Kühn, A. A., Tsui, A., Aziz, T., Ray, N., Brücke, C., Kupsch, A., ... Brown, P. (2009). Pathological synchronisation in the subthalamic nucleus of patients with Parkinson's disease relates to both bradykinesia and rigidity. Experimental Neurology, 215(2), 380-387. https://doi.org/10.1016/j.expneurol.2008.11.008

Labounek, R., Bridwell, D. A., Mareček, R., Lamoš, M., Mikl, M., Slavíček, T., ... Jan, J. (2018). Stable scalp EEG spatiospectral patterns across paradigms estimated by group ICA. Brain Topography, 31(1), 76-89. https://doi.org/10.1007/s10548-017-0585-8

Leuchter, A. F., Cook, I. A., Gilmer, W. S., Marangell, L. B., Burgoyne, K. S., Howland, R. H., ... Greenwald, S. (2009). Effectiveness of a quantitative electroencephalographic biomarker for predicting differential response or remission with escitalopram and bupropion in major depressive disorder. Psychiatry Research, 169(2), 132-138. https://doi.org/10.1016/j.psychres.2009.04.004

Leuchter, A. F., Cook, I. A., Marangell, L. B., Gilmer, W. S., Burgoyne, K. S., Howland, R. H., ... Greenwald, S. (2009). Comparative effectiveness of biomarkers and clinical indicators for predicting outcomes of SSRI treatment in major depressive disorder: Results of the BRITE-MD study. Psychiatry Research, 169(2), 124-131. https://doi.org/10.1016/j.psychres.2009.06.004

Leuchter, A. F., Hunter, A. M., Jain, F. A., Tartter, M., Crump, C., \& Cook, I. A. (2017). Escitalopram but not placebo modulates brain rhythmic oscillatory activity in the first week of treatment of major depressive disorder. Journal of Psychiatric Research, 84, 174-183. https://doi.org/10.1016/j.jpsychires.2016.10.002

Li, C.-T., Hsieh, J.-C., Huang, H.-H., Chen, M.-H., Juan, C.-H., Tu, P.-C., ... Su, T.-P. (2016). Cognition-modulated frontal activity in prediction and augmentation of antidepressant efficacy: A randomized controlled pilot study. Cerebral Cortex, 26(1), 202-210. https:// doi.org/10.1093/cercor/bhu191

Light, G. A., Swerdlow, N. R., Thomas, M. L., Calkins, M. E., Green, M. F., Greenwood, T. A., ... Turetsky, B. I. (2015). Validation of mismatch negativity and P3a for use in multi-site studies of schizophrenia: Characterization of demographic, clinical, cognitive, and functional correlates in COGS-2. Schizophrenia Research, 163(13), 63-72. https://doi.org/10.1016/j.schres.2014.09.042

Lorenzo-Seva, U., \& Berge, J. M. F. (2006). Tucker' s congruence coefficient as a meaningful index of factor similarity. Methodology, 2(2), 57-64. https://doi.org/10.1027/1614-2241.2.2.57

Martín-Buro, M. C., Garcés, P., \& Maestú, F. (2016). Test-retest reliability of resting-state magnetoencephalography power in sensor and source space. Human Brain Mapping, 37(1), 179-190. https:// doi.org/10.1002/hbm.23027

Mayberg, H. S. (1997). Limbic-cortical dysregulation: a proposed model of depression. Journal of Neuropsychiatry and Clinical Neurosciences, 9(3), 471-481. https://doi.org/10.1176/jnp.9.3.471

Mcmenamin, B. W., Shackman, A. J., Maxwell, J. S., Bachhuber, D. R. W., Koppenhaver, A. M., Greischar, L. L., \& Davidson, R. J. (2010).
Validation of ICA-based myogenic artifact correction for scalp and source-localized EEG. NeuroImage, 49(3), 2416-2432. https://doi. org/10.1016/j.neuroimage.2009.10.010

Meehl, P. E. (1995). Bootstraps taxometrics: Solving the classification problem in psychopathology. American Psychologist, 50, 266-275. https://doi.org/10.1037/0003-066X.50.4.266

Michel, C. M., Murray, M. M., Lantz, G., Gonzalez, S., Spinelli, L., \& Grave De Peralta, R. (2004). EEG source imaging. Clinical Neurophysiology, 115(10), 2195-2222. https://doi.org/10.1016/j. clinph.2004.06.001

Monti, M. M., Vanhaudenhuyse, A., Coleman, M. R., Boly, M., Pickard, J. D., Tshibanda, L., ... Laureys, S. (2010). Willful modulation of brain activity in disorders of consciousness. New England Journal of Medicine, 362(7), 579-589. https://doi.org/10.1056/NEJMoa0905370

Mulert, C., Juckel, G., Brunnmeier, M., Karch, S., Leicht, G., Mergl, R., ... Pogarell, O. (2007). Rostral anterior cingulate cortex activity in the theta band predicts response to antidepressive medication. Clinical EEG and Neuroscience, 38(2), 78-81. https://doi. org/10.1177/155005940703800209

Näpflin, M., Wildi, M., \& Sarnthein, J. (2008). Test-retest reliability of EEG spectra during a working memory task. NeuroImage, 43(4), 687-693. https://doi.org/10.1016/j.neuroimage.2008.08.028

Nunez, P. L., Nunez, M. D., \& Srinivasan, R. (2019). Multi-scale neural sources of EEG: Genuine, equivalent, and representative. A Tutorial Review. Brain Topography, 32(2), 193-214. https://doi. org/10.1101/391318

Nunez, P. L., \& Srinivasan, R. (2006). Electric fields of the brain: The neurophysics of EEG. New York, NY: Oxford University Press.

Olbrich, S., Mulert, C., Karch, S., Trenner, M., Leicht, G., Pogarell, O., \& Hegerl, U. (2009). EEG-vigilance and b effect during simultaneous EEG/fMRI measurement. NeuroImage, 45(2), 319-332. https:// doi.org/10.1016/j.neuroimage.2008.11.014

Olvet, D. M., \& Hajcak, G. (2009). The stability of error-related brain activity with increasing trials. Psychophysiology, 46, 957-961. https://doi.org/10.1111/j.1469-8986.2009.00848.x

Onton, J., Delorme, A., \& Makeig, S. (2005). Frontal midline EEG dynamics during working memory. NeuroImage, 27, 341-356. https:// doi.org/10.1016/j.neuroimage.2005.04.014

Parra, L., \& Sajda, P. (2003). Blind source separation via generalized eigenvalue decomposition. The Journal of Machine Learning Research, 4, 1261-1269. Retrieved from http://dl.acm.org/citation. cfm?xml:id=945365.964305

Parra, L. C., Spence, C. D., Gerson, A. D., \& Sajda, P. (2005). Recipes for the linear analysis of EEG. NeuroImage, 28(2), 326-341. https:// doi.org/10.1016/j.neuroimage.2005.05.032

Pascual-Marqui, R. D. (2007). Discrete, 3D distributed, linear imaging methods of electric neuronal activity. Part 1: exact, zero error localization. ArXiv, 1-16. Retrieved from http://arxiv.org/abs/0710.3341

Pascual-Marqui, R. D., Faber, P. L., Kinoshita, T., Kochi, K., Milz, P., Nishida, K., \& Yoshimura, M. (2018). Comparing EEG/MEG neuroimaging methods based on localization error, false positive activity, and false positive connectivity. bioRxiv, 269753.

Pascual-Marqui, R. D., Michel, C. M., \& Lehmann, D. (1994). Low resolution electromagnetic tomography: A new method for localizing electrical activity in the brain. International Journal of Psychophysiology, 18(1), 49-65. https://doi. org/10.1016/0167-8760(84)90014-X

Patrick, C. J., \& Hajcak, G. (2016). RDoC: Translating promise into progress. Psychophysiology, 53(3), 415-424. https://doi. org/10.1111/psyp.12612 
Perrin, F., Pernier, J., Bertrand, O., \& Echallier, J. F. (1989). Spherical splines for scalp potential and current density mapping. Electroencephalography and Clinical Neurophysiology, 72(2), 184187. https://doi.org/10.1016/0013-4694(89)90180-6

Perrin, F., Pernier, J., Bertrand, O., \& Echallier, J. F. (1990). Corrigenda. Electroencephalography and Clinical Neurophysiology, 76, 565566. https://doi.org/10.1016/0013-4694(90)90009-9

Pizzagalli, D. A. (2011). Frontocingulate dysfunction in depression: Toward biomarkers of treatment response. Neuropsychopharmacology, 36(1), 183-206. https://doi.org/10.1038/npp.2010.166

Pizzagalli, D. A., Oakes, T. R., \& Davidson, R. J. (2003). Coupling of theta activity and glucose metabolism in the human rostral anterior cingulate cortex: an EEG/PET study of normal and depressed subjects. Psychophysiology, 40(6), 939-949. https://doi. org/10.1111/1469-8986.00112

Pizzagalli, D., Pascual-Marqui, R. D., Nitschke, J. B., Oakes, T. R., Larson, C. L., Abercrombie, H. C., ... Davidson, R. J. (2001). Anterior cingulate activity as a predictor of degree of treatment response in major depression: Evidence from brain electrical tomography analysis. The American Journal of Psychiatry, 158(3), 405-415. Retrieved from http://www.ncbi.nlm.nih.gov/pubmed/11229981. https://doi.org/10.1176/appi.ajp.158.3.405

Pizzagalli, D. A., Webb, C. A., Dillon, D. G., Tenke, C. E., Kayser, J., Goer, F., ... Trivedi, M. H. (2018). Pretreatment rostral anterior cingulate cortex theta activity in relation to symptom improvement in depression: A randomized clinical trial. JAMA Psychiatry, 75(6), 547-554. https://doi.org/10.1001/jamapsychiatry.2018.0252

Popov, T., Popova, P., Harkotte, M., Awiszus, B., Rockstroh, B., \& Miller, G. A. (2018). Cross-frequency interactions between frontal theta and posterior alpha control mechanisms foster working memory. NeuroImage, 181, 728-733. https://doi.org/10.1016/j.neuro image.2018.07.067

Rentzsch, J., Adli, M., Wiethoff, K., Gómez-Carrillo de Castro, A., Gallinat, J. (2014). Pretreatment anterior cingulate activity predicts antidepressant treatment response in major depressive episodes. European Archives of Psychiatry and Clinical Neuroscience, 264(3), 213-223. https://doi.org/10.1007/s00406-013-0424-1

Sadaghiani, S., \& Kleinschmidt, A. (2016). Brain networks and $\alpha$-oscillations: Structural and functional foundations of cognitive control. Trends in Cognitive Sciences, 20(11), 805-817. https://doi. org/10.1016/j.tics.2016.09.004

Sauseng, P., Griesmayr, B., Freunberger, R., \& Klimesch, W. (2010). Control mechanisms in working memory: A possible function of EEG theta oscillations. Neuroscience and Biobehavioral Reviews, 34(7), 1015-1022. https://doi.org/10.1016/j.neubiorev. 2009.12.006

Schacter, D. (1977). EEG theta waves and physiological phenomena: A review and analysis. Biological Psychology, 5(1), 47-82. https://doi. org/10.1016/0301-0511(77)90028-X

Scharf, F., \& Nestler, S. (2018). Principles behind variance misallocation in temporal exploratory factor analysis for ERP data: Insights from an inter-factor covariance decomposition. International Journal of Psychophysiology, 128, 119-136. https://doi.org/10.1016/j.ijpsy cho.2018.03.019

Scheeringa, R., Bastiaansen, M. C. M., Petersson, K. M., Oostenveld, R., Norris, D. G., \& Hagoort, P. (2008). Frontal theta EEG activity correlates negatively with the default mode network in resting state. International Journal of Psychophysiology, 67(3), 242-251. https:// doi.org/10.1016/j.ijpsycho.2007.05.017
Schmidt, F. M., Sander, C., Dietz, M.-E., Nowak, C., Schröder, T., Mergl, R., ... Hegerl, U. (2017). Brain arousal regulation as response predictor for antidepressant therapy in major depression. Scientific Reports, 7, 1-10. https://doi.org/10.1038/srep45187

Shackman, A., McMenamin, B., Maxwell, J., Greischar, L., \& Davidson, R. J. (2010). Identifying robust and sensitive frequency bands for interrogating neural oscillations. NeuroImage, 51(4), 1319-1333. https://doi.org/10.1016/j.neuroimage.2010.03.037

Smith, E. E., Cavanagh, J. F., \& Allen, J. J. B. B. (2018). Intracranial source activity (eLORETA) related to scalp-level asymmetry scores and depression status. Psychophysiology, 55(1), 1-16. https://doi. org/10.1111/psyp.13019

Smith, E. E., Reznik, S. J., Stewart, J. L., \& Allen, J. J. B. (2017). Assessing and conceptualizing frontal EEG asymmetry: An updated primer on recording, processing, analyzing, and interpreting frontal alpha asymmetry. International Journal of Psychophysiology, 111, 98-114. https://doi.org/10.1016/j.ijpsycho.2016.11.005

Sockeel, S., Schwartz, D., Pélégrini-issac, M., \& Benali, H. (2016). Large-scale functional networks identified from resting-state EEG using spatial ICA. PLoS ONE, 1-18, https://doi.org/10.5061/dryad. v9f16

Stewart, J. L., Coan, J. A., Towers, D. N., \& Allen, J. J. B. (2011). Frontal EEG asymmetry during emotional challenge differentiates individuals with and without lifetime major depressive disorder. Journal of Affective Disorders, 129(1-3), 167-174. https://doi.org/10.1016/ j.jad.2010.08.029

Strijkstra, A. M., Beersma, D. G. M., Drayer, B., Halbesma, N., \& Daan, S. (2003). Subjective sleepiness correlates negatively with global alpha $(8-12 \mathrm{~Hz})$ and positively with central frontal theta (4-8 Hz) frequencies in the human resting awake electroencephalogram. Neuroscience Letters, 340(1), 17-20. https://doi.org/10.1016/ S0304-3940(03)00033-8

Tenke, C. E., \& Kayser, J. (2005). Reference-free quantification of EEG spectra: Combining current source density (CSD) and frequency principal components analysis (fPCA). Clinical Neurophysiology, 116(12), 2826-2846. https://doi.org/10.1016/j. clinph.2005.08.007

Tenke, C. E., \& Kayser, J. (2012). Generator localization by current source density (CSD): Implications of volume conduction and field closure at intracranial and scalp resolutions. Clinical Neurophysiology, 123(12), 2328-2345. https://doi.org/10.1016/ j.clinph.2012.06.005

Tenke, C. E., \& Kayser, J. (2015). Surface Laplacians (SL) and phase properties of EEG rhythms: Simulated generators in a volumeconduction model. International Journal of Psychophysiology, 97(3), 285-298. https://doi.org/10.1016/j.ijpsycho.2015.05.008

Tenke, C. E., Kayser, J., Abraham, K., Alvarenga, J. E., \& Bruder, G. E. (2015). Posterior EEG alpha at rest and during task performance: Comparison of current source density and field potential measures. International Journal of Psychophysiology, 97(3), 299-309. https:// doi.org/10.1016/j.ijpsycho.2015.05.011

Tenke, C. E., Kayser, J., Manna, C. G., Fekri, S., Kroppmann, C. J., Schaller, J. D., ... Bruder, G. E. (2011). Current source density measures of electroencephalographic alpha predict antidepressant treatment response. Biological Psychiatry, 70(4), 388-394. https://doi. org/10.1016/j.biopsych.2011.02.016

Tenke, C. E., Kayser, J., Pechtel, P., Webb, C. A., Dillon, D. G., Goer, F., ... Trivedi, M. (2017). Demonstrating test-retest reliability of electrophysiological measures for healthy adults in a 
multisite study of biomarkers of antidepressant treatment response. Psychophysiology, 54(1), 34-50. https://doi.org/10.1111/ psyp. 12758

Trivedi, M. H., Mcgrath, P. J., Fava, M., Parsey, R. V., Benji, T., Phillips, M. L., ... Weissman, M. M. (2016). Establishing moderators and biosignatures of antidepressant response in clinical care (EMBARC): Rationale and design. Journal of Psychiatric Research, 78, 11-23. https://doi.org/10.1016/j.jpsyc hires.2016.03.001.ESTABLISHING

Ulke, C., Tenke, C. E., Kayser, J., Sander, C., Bottger, D., Wong, L. Y. X., ... Bruder, G. E. (2019). Resting EEG Measures of Brain Arousal in a Multisite Study of Major Depression. Clinical EEG and Neuroscience, 50(1), 3-12. https://doi.org/10.1177/15500 59418795578

Ulrich, G., Renfordt, E., \& Frick, K. (1986). The topographical distribution of alpha-activity in the resting EEG of endogenousdepressive in-patients with and without clinical response to pharmacotherapy. Pharmacopsychiatry, 19(4), 272-273. https://doi. org/10.1055/s-2007-1017230

Ulrich, G., Renfordt, E., Zeller, G., \& Frick, K. (1984). Interrelation between changes in the EEG and psychopathology under pharmacotherapy for endogenous depression. Pharmacopsychiatry, 17(6), 178-183. https://doi.org/10.1055/s-2007-1017433

Vidaurre, D., Hunt, L. T., Quinn, A. J., Hunt, B. A. E. E., Brookes, M. J., Nobre, A. C., ... Quinn, A. J. (2018). Spontaneous cortical activity transiently organises into frequency specific phase-coupling networks. Nature Communications, 9(1), 2987. https://doi.org/10.1038/ s41467-018-05316-z

Wade, E. C., \& Iosifescu, D. V. (2016). Using electroencephalography for treatment guidance in major depressive disorder. Biological Psychiatry: Cognitive Neuroscience and Neuroimaging, 1(5), 411422. https://doi.org/10.1016/j.bpsc.2016.06.002

Waters, A. C., \& Mayberg, H. S. (2017). Brain-based biomarkers for the treatment of depression: Evolution of an idea. Journal of the
International Neuropsychological Society, 23(9-10), 870-880. https://doi.org/10.1017/s1355617717000881

Weinberg, A., Riesel, A., \& Hajcak, G. (2012). Integrating multiple perspectives on error-related brain activity: The ERN as a neural indicator of trait defensive reactivity. Motivation and Emotion, 36(1), 84-100. https://doi.org/10.1007/s11031-011-9269-y

Westmoreland, B. F., \& Klass, D. W. (1986). Midline theta rhythm. Archives of Neurology, 43(2), 139-141. https://doi.org/10.1001/ archneur.1986.00520020033013

Whitton, A. E., Webb, C. A., Dillon, D. G., Kayser, J., Rutherford, A., Goer, F., ... \& Adams, P. (2019). Pretreatment Rostral Anterior Cingulate Cortex Connectivity With Salience Network Predicts Depression Recovery: Findings From the EMBARC Randomized Clinical Trial. Biological Psychiatry, 85(10), 872-880. https://doi. org/10.1016/j.biopsych.2018.12.007

Widge, A. S., Bilge, M. T., Montana, R., Chang, W., Rodriguez, C. I., Deckersbach, T., ... Nemeroff, C. B. (2018). Electroencephalographic biomarkers for treatment response prediction in major depressive illness: A meta-analysis. American Journal of Psychiatry, 176, 44-56. https://doi.org/10.1176/appi.ajp.2018.17121358

\section{SUPPORTING INFORMATION}

Additional supporting information may be found online in the Supporting Information section at the end of the article.

How to cite this article: Smith EE, Tenke CE, Deldin PJ, et al. Frontal theta and posterior alpha in resting EEG: A critical examination of convergent and discriminant validity. Psychophysiology. 2020;57:e13483. https://doi.org/10.1111/psyp.13483 